\title{
Alexander Sokolicek \\ Johanna Auinger \\ Ein späthellenistisches Grabrelief aus der Nekropole am Ostabhang des Panayır Dağ
}

Am Ostabhang des Panayır Dağ konnten im Frühjahr 2005 die Fundamente und das teilweise bis in das Aufgehende erhaltene Mauerwerk dreier bislang unbekannter Grabhäuser (1-3) gereinigt und dokumentiert werden. An das Grabhaus 3 schlossen sich zwei Gräber mit Urnenbestattungen an, wobei Grab 1 mit einem späthellenistischen Grabrelief abgedeckt war.

\section{Bericht über die Ausgrabung 2005}

Im Mai des Jahres 2005 wurde am Ostabhang des Panayır Dağ (Abb. 1) westlich der Strecke zwischen Kathodos IV und V der Damianosstoa ${ }^{2}$ eine Fläche von ca. $50 \times 10 \mathrm{~m}$ gereinigt und teilweise freigelegt (Abb. 2). Die Erweiterung der Grabungsfläche nach Osten wurde durch einen modernen Weingarten behindert, der an das Areal anschließt (Abb. 2). Der westliche, dem Panayır Dağ zugewandte Teil besteht aus einer großteils geglätteten und nur teilweise von Erde bedeckten Felsfläche, die relativ abrupt oberhalb des Weingartens endet.

In diesem Areal ließen Quaderstrukturen, die an der Oberfläche sichtbar waren, auf Grabhäuser der Nekropole, die sich am östlichen Abhang des Panayır Dağ erstreckt, schließen³. Diese Nekropole wurde erstmals von J. T. Wood erwähnt ${ }^{4}$. Von Begehungen durch J. Keil ${ }^{5}$ und von Arbeiten F. Miltners ${ }^{6}$ abgesehen, wurde sie jedoch nie systematisch untersucht, wie die Nekropolen von Ephesos im Allgemeinen nahezu unbekannt sind ${ }^{7}$.

Zeugnis für die Nekropole am Ostabhang des Panayır Dağ legen zahlreiche Grabinschriften ${ }^{8}$ und Grabreliefs ${ }^{9}$. Grabstätten und Sarkophage, die eine Nutzung der Prozessionsstraße entlang der Trasse der Damia-

\footnotetext{
${ }^{1}$ Die Grabungen fanden vom 2.-5. 5. 2005 unter der örtlichen Leitung von A. Sokolicek statt. Für die Publikationserlaubnis sei dem Leiter der Ausgrabungen von Ephesos, F. Krinzinger, gedankt. Ferner sei folgenden Personen (alle Wien) gedankt: K. Grossschmidt und F. Kanz (anthropologische Bestimmung des Knochenmaterials), Ch. Kurtze (Plan Ephesos - Abb. 1), S. Ladstätter (Keramikanalyse), M. Aurenhammer und E. Rathmayr (Anregungen und Korrekturen). - Abkürzungen und Zitierweisen folgen den Richtlinien der ÖJh 69, 2000, 357 ff., die hier zusätzlich verwendeten Abkürzungen finden sich am Ende des Beitrags.

${ }^{2}$ Dieser Bereich liegt westlich der Sarkophage, die im Plan bei Pietsch (1999) Taf. 93 nördlich von K(athodos) IV eingetragen sind; zur Lokalisation s. dazu die Bemerkung in Pietsch (1999) 457 Anm. 17 (»zwischen Abschnitt K IV und K V«). Zu diesen Sarkophagen s. u. Anm. 23. Die Bergung der Sarkophage fand im Frühjahr 2005 durch M. Steskal (Wien) statt.

3 Zuletzt zu dieser Nekropole: Pietsch (1999).

${ }^{4}$ Wood (1877) $113 \mathrm{ff}$.

5 J. Keil, Grabbau mit Unterweltssarkophag aus Ephesos, ÖJh 17, 1914, 133 ff.; J. Keil, XV. Vorläufiger Bericht über die Ausgrabungen in Ephesos, ÖJh 26, 1930, Beibl. 7 ff.; s. auch RE Suppl. XII (1970) 1677-1678 s. v. Ephesos (W. Alzinger).

${ }^{6}$ F. Miltner, XXII. Vorläufiger Bericht über die Ausgrabungen in Ephesos, ÖJh 44, 1959, Beibl. 309.

7 Die Nekropolen sind nur in Ausschnitten durch Zufallsfunde bekannt, systematische Untersuchungen haben lediglich im Rahmen allgemeiner Surveys stattgefunden (s. etwa J. Keil, ÖJh 15, 1912, Beibl. 185 ff. zur Nekropole beim Hermaion sowie die Karten von A. Schindler 1897 [ÖAI Inv. 2010/1a, abgebildet bei St. Groh, Die Topographie der Oberstadt von Ephesos. Vorbericht über das Projektjahr 2000, ÖJh 70, 2001, 25 Abb. 4] bzw. von Wood [1877] Abb. 39; ferner Wood [1877] 111 ff.). Allgemein dazu s. Pietsch (1999) 455 f. Auch von anderen großen Städten in Westkleinasien sind die Nekropolen kaum bekannt, s. dazu Berns (2003) Anm. 113 und 114 mit Bibliographie.

${ }^{8}$ IvE 1672-1677. 2100-2158.

${ }^{9}$ Atalay (1973) 231 ff. Ein weiteres Grabrelief vom Ostabhang des Panayır Dağ bei Atalay (1973) 231 ff. bes. 242 ff. mit Abb. 6; Atalay (1988) 47 f. Nr. 17 (mit älterer Bibliographie).
} 


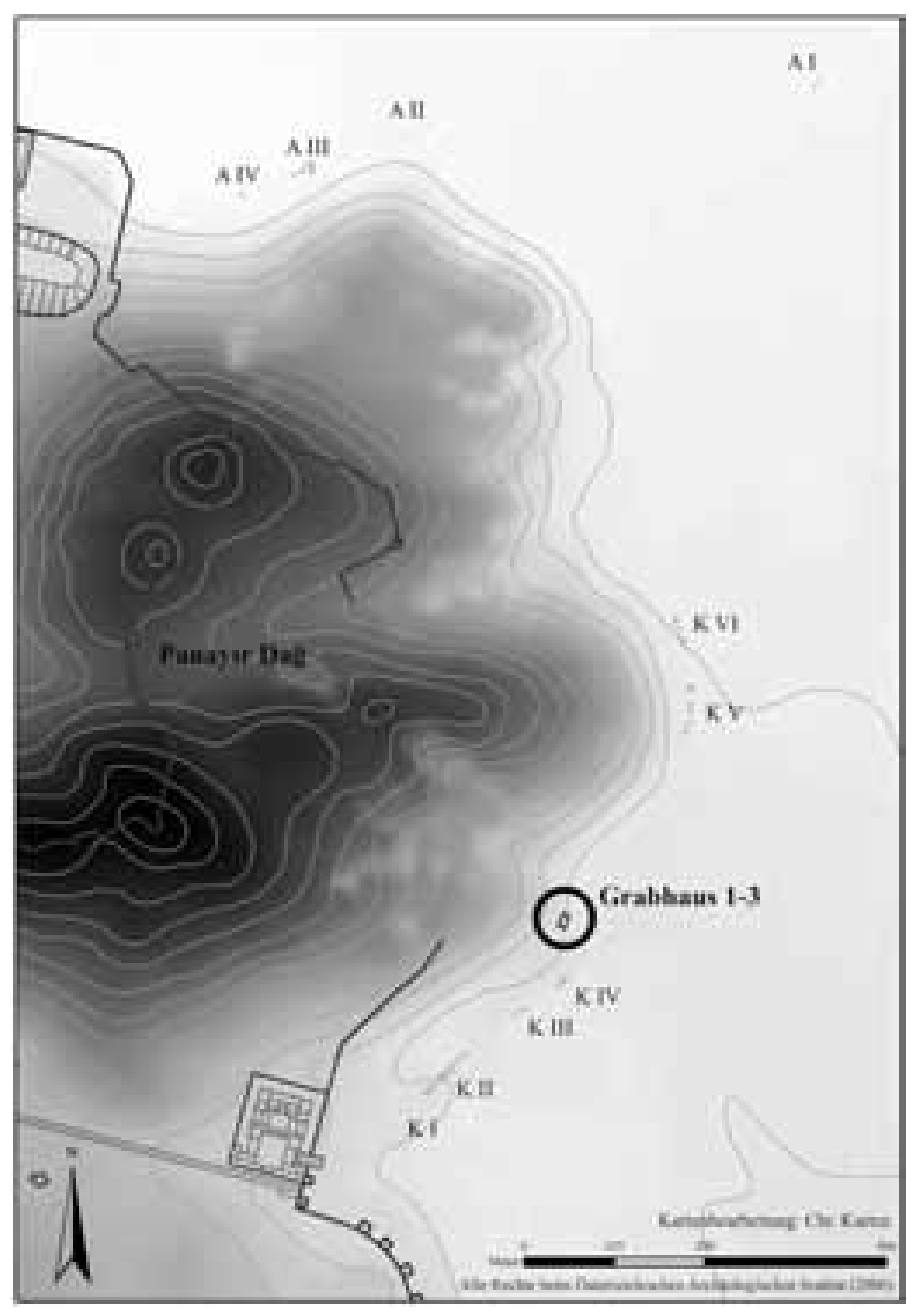

1 Bereich der Nekropole am Osthang des Panayır Dağ

nosstoa zwischen dem Artemision und der hellenistisch-römischen Stadt ab augusteischer Zeit belegen ${ }^{10}$, sind auch im Zuge der Forschungen an der Damianosstoa durch D. Knibbe und H. Thür bekannt geworden ${ }^{11}$. Die außerhalb ihres ursprünglichen Zusammenhangs relativ zahlreich gefundenen Grabreliefs weisen jedoch deutlich auf die Nutzung dieses Areals als Nekropole seit hellenistischer Zeit hin $^{12}$. Die genaue Ausdehnung der Gräberfelder am Ostabhang des Panayır Dağ ist nicht bekannt, dürfte sich aber weit über die Damianosstoa hinaus entlang der Straße nach Magnesia am Mäander erstreckt haben ${ }^{13}$.

Das Ziel der zeitlich und räumlich beschränkten Arbeiten am Fuß des Panayır Dağ ${ }^{14}$ lag in der Freilegung und Klärung der an der Oberfläche zutage getretenen Quaderstrukturen (Abb. 2). Die Reste gehören zu insgesamt drei Grabhäusern (Abb. 3) ${ }^{15}$. Die beiden im Grabungsareal nördlich situierten Grabhäuser (1 und 2) liegen unterhalb des Felsabbruchs und bestehen aus kleinteiligen Kalksteinen mit Mörtelbindung ${ }^{16}$. Die Grabhäuser 1 und 2 sind seitlich miteinander verbunden und waren ursprünglich von Tonnen überwölbt, die sich im Ansatz erhalten haben (Abb. 4). Das besser erhaltene Grabhaus 2 ist gegenüber dem nördlich anschließenden Grabhaus 1 etwas nach Osten vorgerückt, weshalb die Fassaden der beiden Grabhäuser nicht bündig abschließen. Wegen des benachbarten Weingartens, der direkt an Grabhaus 2 angrenzt, konnte dessen Fassade nicht freigelegt werden; wahrscheinlich wurde sie durch die Feldarbeit im Weingarten zerstört ${ }^{17}$. Die Längsmauern des Grabhauses verlieren sich nach etwa $1 \mathrm{~m}$ (Nordwestwand) bzw. nach 0,30 m (Südostwand);

\footnotetext{
${ }^{10}$ S. zuletzt Thür (1999) mit Bibliographie.

${ }^{11}$ Knibbe - Langmann (1993) 22 ff.; H. Thür, Zur Bautechnik und Rekonstruktion der Damianusstoa, in: Knibbe - Langmann (1993) 47 ff.; E. Trinkl, Vorbericht über die Versuchsgrabung im Abschnitt IV der Kathodos, in: Knibbe - Langmann (1993) 36 ff.; Pietsch (1999) 455 ff.; D. Knibbe - H. Thür, Via sacra Ephesiaca, 2. Grabungen und Forschungen 1992 und 1993, BerMatÖAI 6 (1995) und bes. W. Pietsch - E. Trink1, Der Grabungsbericht der Kampagnen 1992/93, in: ebenda 19 ff.; D. Knibbe, Via sacra Ephesiaca, in: H. Friesinger - F. Krinzinger (Hrsg.), 100 Jahre Österreichische Forschungen in Ephesos. Akten des Symposions Wien 1995, AForsch 1, DenkschrWien 260 (1999) 449 ff.; Thür (1999).

12 Atalay (1973) 231 f.; E. Atalay, Späthellenistische Grabreliefs aus Ephesos, in: The Proceedings of the 10th International Congress of Classical Archeology, Ankara 1973 (1978) 616; s. u. (Beitrag J. Auinger).

13 s. dazu Wood (1877) 114 ff. und zuletzt Pietsch (1999) 455 f.

${ }^{14}$ Im Osten wird das Grabungsareal - wie erwähnt - von einem Weingarten begrenzt (s. Abb. 2), durch den weitere Freilegungen behindert wurden.

${ }^{15}$ In einer Entfernung von 4,50 m nach Südosten befinden sich drei weitere Blöcke, die den Beginn eines weiteren, nur rudimentär erhaltenen Grabhauses markieren (vgl. Abb. 2).

${ }^{16} 0,10 \times 0,10 \mathrm{~m}-0,30 \times 0,40 \mathrm{~m}$.

${ }_{17}$ Die Längsmauern des Grabhauses 2 schließen im Osten nicht mit gerader Kante ab, sondern sind abgegraben, vgl. Abb. 2 und 3.
} 
die lichte Breite des Grabhauses 2 liegt bei $2,10 \mathrm{~m}$. Mit Sicherheit endet mit diesem Grabhaus die Reihe der tonnenüberwölbten Grabhäuser unterhalb des Felsabbruchs. Das in einer Entfernung von $1,50 \mathrm{~m}$ weiter südlich des Grabhauses 2 gelegene Grabhaus 3 (Abb. 3) kann wegen seiner Lage und seines Grundrisses nicht zu den überwölbten Grabhäusern gehören.

Die Rückwand des Grabhauses 2 hat sich bis zum Scheitelpunkt des Gewölbes $^{18}$ gut erhalten, das Gewölbe selbst reicht - bei einer errechneten Gewölbehöhe von ca. $1 \mathrm{~m}$ - bis etwa 0,70 m oberhalb des Gewölbeansatzes. Die Rückwand

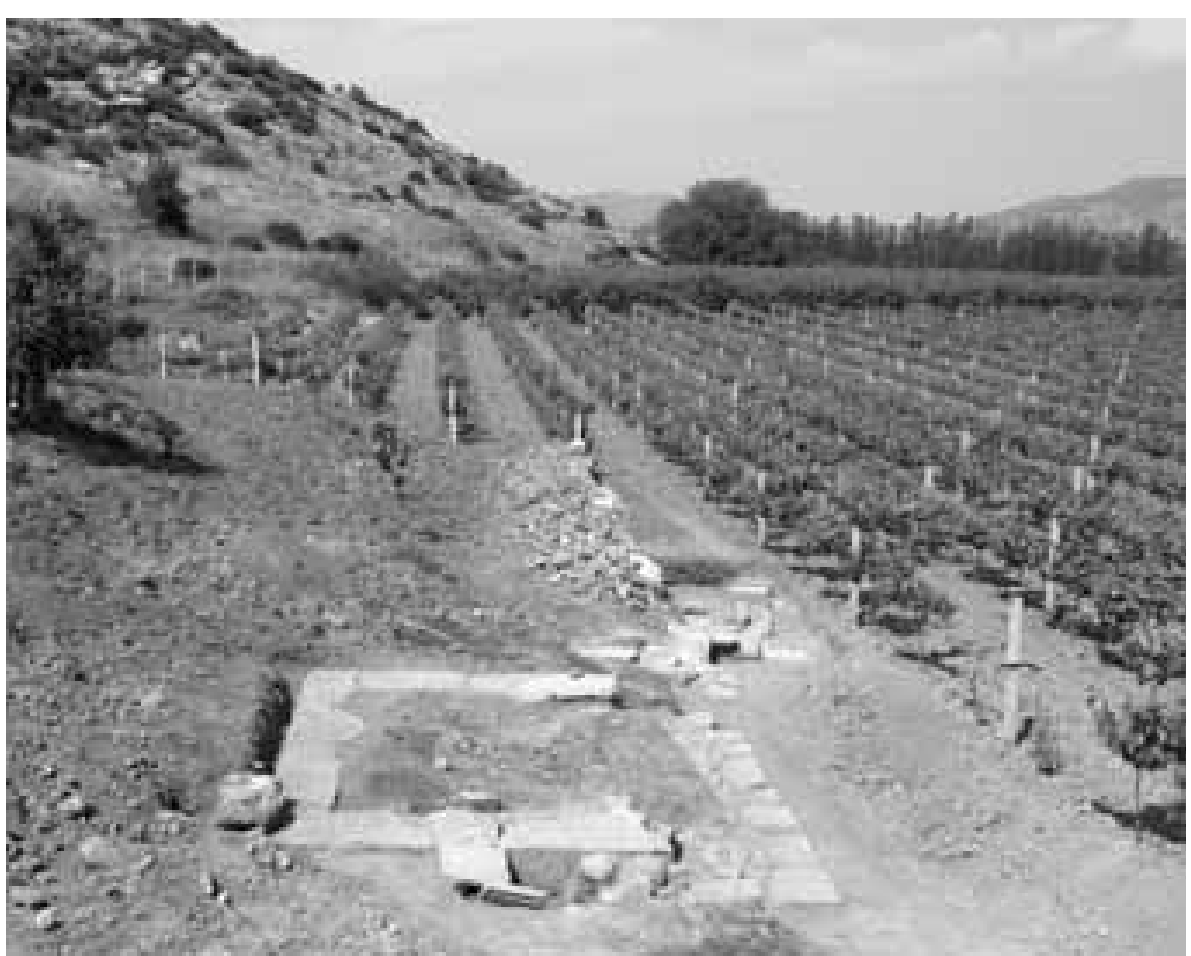

2 Untersuchte Fläche (Blick nach Norden) ist durch drei etwa $0,50 \mathrm{~m}$ breite wie tiefe und etwa $0,60 \mathrm{~m}$ hohe loculi gegliedert, deren oberster mit dem Scheitel des Tonnengewölbes abschließt. Die beiden anderen loculi liegen je seitwärts darunter (Abb. 4).

Von Grabhaus 1 konnte zwar die Fassade, nicht aber die Rückwand freigelegt werden, da sich oberhalb der beiden Grabhäuser 1 und 2 eine moderne Mauer aus großen Feldsteinen befindet, die nicht entfernt werden konnte. Die Fassade ist durch zwei Orthostaten gegliedert (Abb. 3), die wahrscheinlich mittig zum

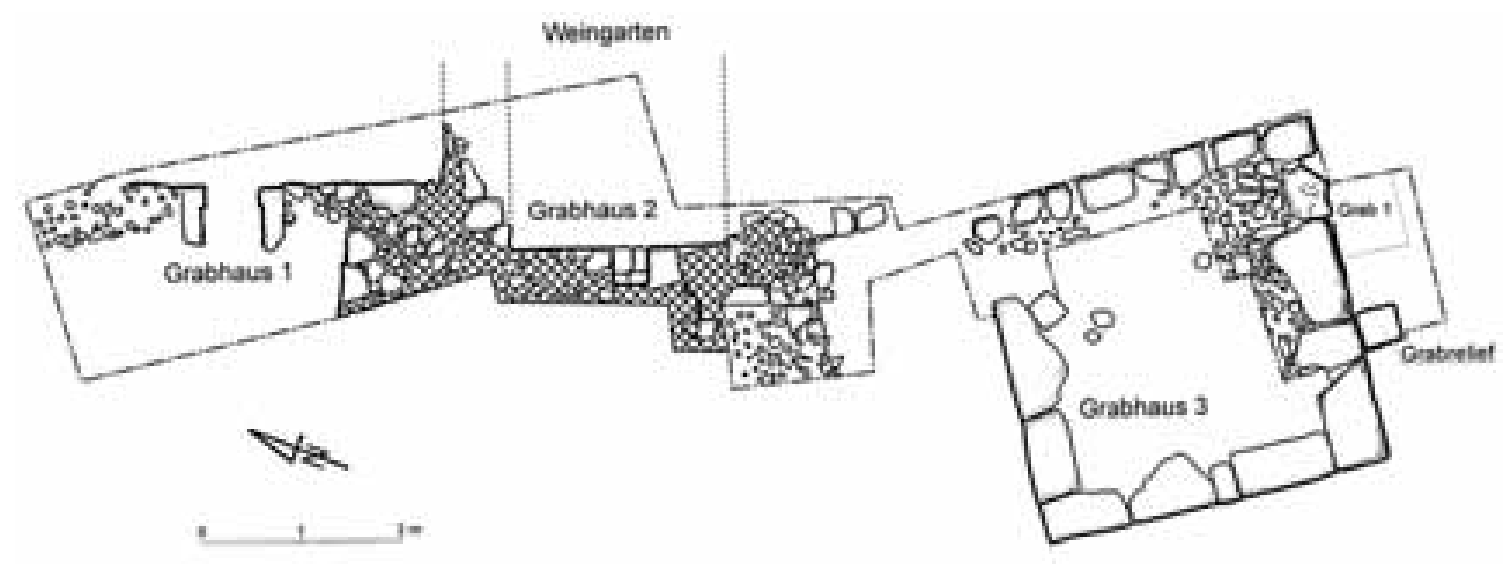

3 Grundrisse der Grabhäuser 1-3

\footnotetext{
${ }^{18}$ OK (absolute H) 17,00 m.
} 




4 Ansicht der Grabhäuser 1 und 2

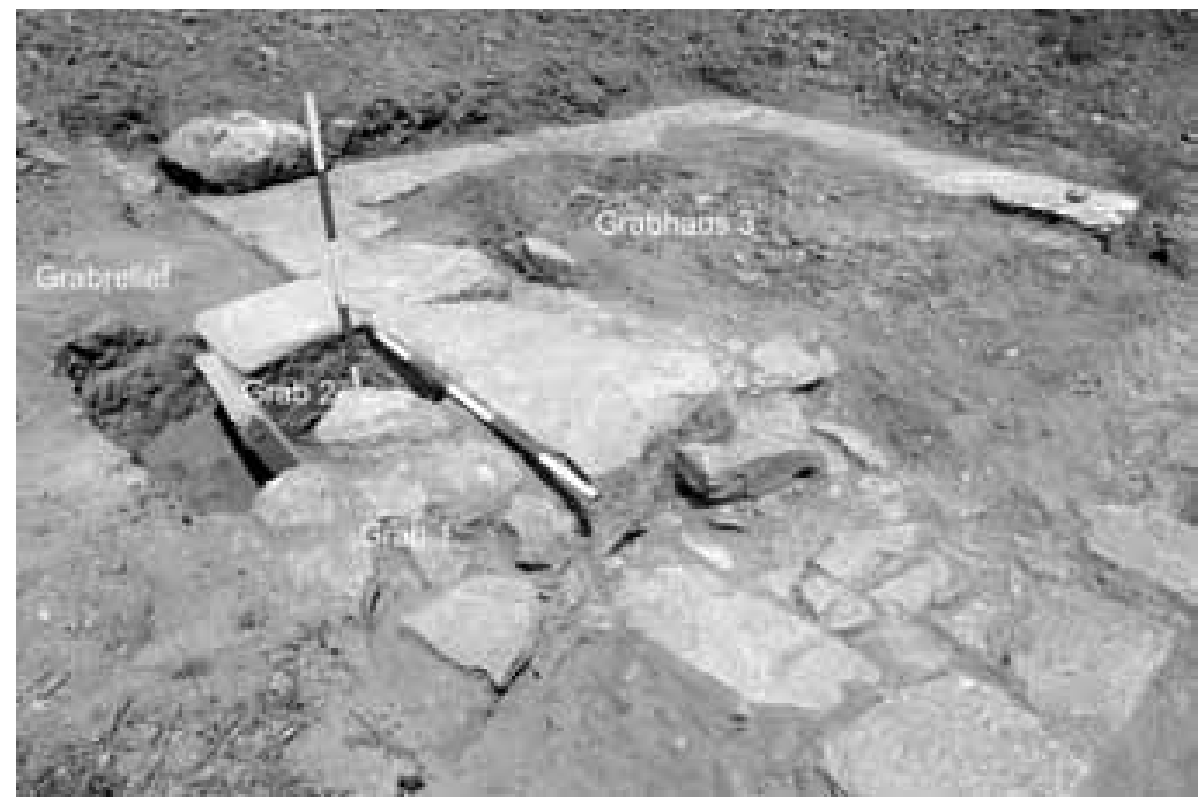

5 Grabhaus 3

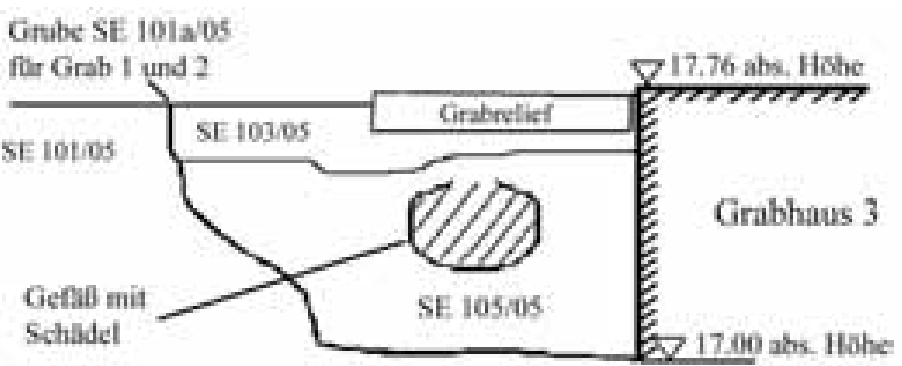


Grabhaus den Eingang ${ }^{19}$ markieren ${ }^{20}$. Von der Fassade hat sich nur die südliche Wand erhalten; der nördliche Teil des Grabhauses 1 ist durch die Feldarbeiten im Bereich des modernen Weingartens erheblich bis gänzlich zerstört worden. Das bis zur zweiten Schar erhaltene Gewölbe von Grabhaus 1 ist mit grobem Putz und einem einfarbigem Feinanstrich versehen ${ }^{21}$.

Derartige Grabhäuser mit Tonnengewölben dürften in den ephesischen Nekropolen, zumindest im Bereich der Damianosstoa, häufig sein: Bereits J. T. Wood berichtet von tonnenüberwölbten Grabbauten ${ }^{22}$, ähnliche Grabkammern wurden 1956 nach dem Hinweis eines Einheimischen von F. Miltner direkt beim Prozessionsweg partiell freigelegt ${ }^{23}$. Eine Datierung der Grabhäuser 1 und 2 lässt sich aus der Architektur nicht ableiten, auch ihre Stratigraphie ist gestört und kann somit keine Anhaltspunkte für eine Datierung geben ${ }^{24}$ : Sie sind aber wohl in Gestalt der von F. Miltner 1956 beim Prozessionsweg untersuchten Grabbauten ${ }^{25} \mathrm{zu}$ rekonstruieren und daher zeitlich mit der Damianosstoa in $\mathrm{Zu}-$ sammenhang zu bringen. Unter wenig aussagekräftigen Funden aus der Aufschüttung, welche die Grabhäuser bedeckte, befand sich eine kleine Bronzeglocke ${ }^{26}$ (Abb. 7), die in unmittelbarer Nähe von Grabhaus 1 gefunden wurde und nach derzeitigem For-

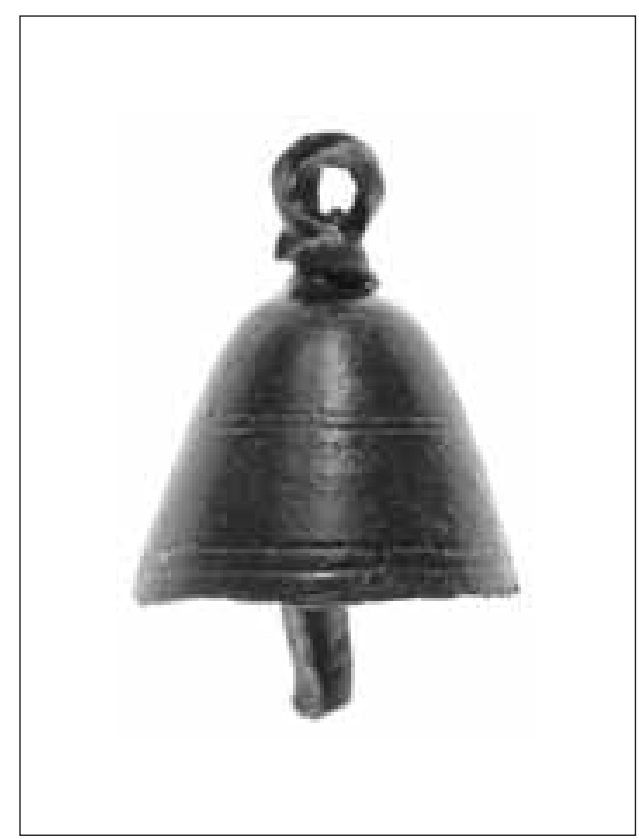

7 Bronzeglocke von Grabhaus 1 schungsstand Mitte 2.-3. Jahrhundert n. Chr. datiert werden $k{ }^{27}$. Die Errichtung dieser überwölbten Grabhäuser dürfte aufgrund der Nähe und des Bezugs zur Damianosstoa gegen das Ende des 2. Jahrhunderts oder in das 3. Jahrhundert n. Chr. fallen.

Gänzlich unterschiedlich ist die Gestalt des Grabhauses 3, das frei stehend auf der erwähnten Felsfläche südwestlich der beiden tonnenüberwölbten Grabhäuser situiert ist (Abb. 3. 5). Es besteht aus großen Kalksteinquadern über einem Kastenfundament mit Bruchstein-Mörtel-Füllung (Abb. 3), von dessen aufgehendem Mauerwerk sich - mit Ausnahme der Nordostseite - die erste Schar erhalten hat ${ }^{28}$. Die Ausrichtung ist gegenüber den Grabhäusern 1 und 2 um etwa $15^{\circ}$ nach Westen gedreht. Grabhaus 3 weist einen quadratischen Grundriss mit einer Seitenlänge von 3,55 m auf.

Die Kalksteinblöcke des Aufgehenden sind bis auf die Innenseite überall gut geglättet, die Seitenkanten exakt ausgearbeitet und mit Kalkmörtel verbunden. Der Steinkasten des Aufgehenden ist wie das Fundament mit einem Bruchstein-Mörtel-Gemisch verfüllt. Ob diese Einfüllung bereits als Rollierung für einen Boden gedient hat, kann nicht entschieden werden. Anhand besser erhaltener Grabbauten ${ }^{29}$ kann man auf eine Sockelzone mit aufgehendem Quadermauerwerk schließen, das sich an einer Seite zur Gräberstraße hin öffnete $^{30}$ - hier wohl an seiner Nordostseite.

Die Einfüllung aus dem Bruchstein-Mörtel-Gemisch sowie die Verwendung von Kalkmörtel bei Grabhaus 3 könnten Anhaltspunkte für die Datierung des Bauwerks geben: Bruchstein-Mörtel-Verbindungen treten in

\footnotetext{
${ }^{19} \mathrm{~B} 0,65 \mathrm{~m}$.

${ }^{20}$ Das Bodenniveau konnte aufgrund der unmittelbaren Nähe des erwähnten Feldes sowie der Bruchsteinmauer nicht abgetieft werden.

${ }^{21}$ Einen derartigen Verputz konnte auch Wood (1877) 121 bei Grabhäusern an der Damianosstoa feststellen.

${ }^{22}$ Wood (1877) 121.

${ }_{23}$ Miltner (Anm. 6) Beibl. 309 und Pietsch (1999) 457 mit Anm. 16.

${ }^{24}$ Aufgrund der Nähe des Feldes und der massiven Bruchsteinmauer war es nicht möglich, gefahrlos tiefer zu graben und das Bodenniveau der Grabhäuser zu erreichen.

${ }^{25}$ s. dazu mit Abb. Pietsch (1999) 457 Taf. 95, 2 und 3.

${ }^{26}$ Fundnr. 1/02.

${ }^{27}$ Für die Datierung danke ich herzlichst I. Kowalleck (Wien).

${ }^{28}$ OK (absolute H) $17,76 \mathrm{~m}$.

29 s. die kubischen Grabbauten in Elaiussa-Sebaste (A. Machatschek, Die Nekropolen und Grabmäler von Elaiussa-Sebaste und Korykos [1967] Kap. 3 A 3 c); allgemein: Berns (2003) 72 ff.

${ }^{30} \mathrm{Zu}$ den Eingangssituationen von Grabhäusern: Berns (2003) $118 \mathrm{ff}$.
} 
Kleinasien im Hellenismus nur selten auf. In der Monumentalarchitektur werden sie erst gegen die Mitte des 1. Jahrhunderts v. Chr. verwendet ${ }^{31}$. Kalkmörtel, ein Bestandteil der Bruchstein-Mörtel-Füllungen, wird in Kleinasien allerdings schon früher angewendet und ist vor allem von Bauwerken in Pergamon, die vor dessen Eingliederung in das Römische Reich 133 v. Chr. datieren, bekannt ${ }^{32}$. In Iasos wurden Grabbauten mit gleicher Einfüllung gefunden, die F. Tomasello ${ }^{33}$ gegen das Ende des ersten Viertels des 1. Jahrhunderts v. Chr. datiert $^{34}$. Der Zeitraum der Einführung von Bruchstein-Mörtel-Füllungen ist allerdings kaum zu erfassen und wahrscheinlich auch regional unterschiedlich ${ }^{35}$.

Nekropolen mit frei stehenden Grabhäusern in lockeren Abständen, zu deren Gattung wohl auch Grabhaus 3 zu zählen ist ${ }^{36}$, sind zumindest für die frühe Kaiserzeit typisch ${ }^{37}$, wohingegen hellenistische Nekropolen in Kleinasien häufig durch Grabbezirke strukturiert sind ${ }^{38}$. Einzelne kubische Grabhäuser, deren Seitenlängen bei kleineren Gebäuden zwischen 3 und $5 \mathrm{~m}$ variieren, treten am Beginn des 1. Jahrhunderts v. Chr. auf ${ }^{39}$. Sie wurden in Quaderbauweise errichtet, wobei für die meisten Grabbauten die Dachgestaltung allerdings nicht mehr zu rekonstruieren ist ${ }^{40}$.

Aufgrund der angewandten Techniken und Ausführung des Grabbaus wird man ihn vorerst in das 1. Jahrhundert v. Chr. setzen, eine genauere Einordnung wegen der erwähnten Problematik der Bruchstein-MörtelFüllung aber nicht treffen können, zumal auch regionale Unterschiede in Kleinasien zu berücksichtigen sind. Gerade Ephesos ist aufgrund der frühen Präsenz von Italikern ab dem 1. Jahrhundert v. Chr. mit der Übernahme ('Akkommodation') technischer Errungenschaften konfrontiert und von Kulturelementen der italischen Halbinsel beeinflusst ${ }^{41}$.

${ }^{31}$ Zur Verwendung von Mörtel im Hellenismus s. H. Lauter, Architektur des Hellenismus (1986) 56 ff.; M. Waelkens, The Adoption of Roman Building Techniques in the Architecture of Asia Minor, in: S. Macready - F. H. Thompson (Hrsg.), Roman Architecture in the Greek World (1987) $94 \mathrm{f}$.

32 A. Conze, Stadt und Landschaft, AvP I 2 (1913) 154. 243 ff.; E. Boehringer - F. Krauss, Der Temenos für den Herrscherkult, AvP IX (1937) 50 f. 76 und allgemein Lauter (Anm. 31) 56 ff. zur Verwendung von Kalkmörtel im Hellenismus.

${ }^{33}$ F. Tomasello, L'aquedotto romano e la necropoli presso l'istmo, Missione archeologica italiana di Iasos II (1991) $161 \mathrm{ff}$. Taf. $29 b$.

${ }^{34}$ Zur Diskussion s. Berns (2003) 221 f., der sich gegen eine Frühdatierung des Grabes Nr. 49 in Iasos ausspricht.

${ }^{35} \mathrm{Zu}$ dieser Problematik s. Waelkens (Anm. 31) 94 f. sowie in Bezug auf die Architektur von Grabmälern Berns (2003) 7 f. und 60. 211, wo sich Berns generell gegen eine frühere Datierung von Bruchstein-Mörtel-Füllung vor der frühen Kaiserzeit ausspricht; S. 186 mit Verweis auf die allgemein unsichere Datierungsmöglichkeit.

${ }^{36}$ Von der Ostseite des Panayır Dağ sind auch andere frei stehende Grabhäuser bekannt, s. Knibbe - Langmann (1993); Thür (1999).

37 Anemourion: E. Alföldi-Rosenbaum, Anamur-Nekropolü (The Necropolis of Anemurium) (1971) 91 ff. 121 ff. - Zur Orientierung und Vorbildern s. Berns (2003) 112 ff. 132 ff. In Italien sind frei stehende Grabhäuser in den Nekropolen erst ab der Mitte des 1. Jhs. n. Chr. üblich: H. v. Hesberg, Römische Grabbauten (1992) 38 ff. 42 ff.

${ }^{38}$ Berns (2003) 10 ff. (mit Bibliographie). Zu den Nekropolen von Alexandria und Samos s. Schmidt (1991) 4 ff. mit Bibliographie; Fabricius (1999) 105. 143 ff. (Samos). Einzelne Grabmalformen sind bei H. Lauter-Bufe, Zu einem hellenistischen Grabzeichen aus Rhodos, AM 98, 1983, 155 ff., H. v. Hesberg, Bildsyntax und Erzählweisen in der hellenistischen Flächenkunst, JdI 103, 1988, 320 ff. und Pfanner (1989) 185 angeführt. Zur Nekropole auf Rheneia bei Delos s. D. W. v. Moock, Delische 'Rundaltäre'. Zur Ausstattung hellenistischer Grabbezirke auf Rheneia, AM 119, 2004, 373 f. mit Bibliographie. Zu den Nekropolen und Bestattungsweisen in Samos, Rhodos und Kyzikos im sozialen Kontext s. Fabricius (1999) 105. 143 ff. (Samos); 105.207 ff. (Rhodos); 314 ff. (Kyzikos).

39 Zu den Maßen und zur Gestalt späthellenistisch-frühkaiserzeitlicher Grabhäuser von Ephesos und Elaiussa-Sebaste s. Berns (2003) 72 ff. 112 ff. und allgemein 140 ff. Vgl. ferner die Anlagen der Grabhäuser in Elaiussa-Sebaste aus der 1. Hälfte des 1. Jhs. n. Chr.: Machatschek (Anm. 29) 74: Seitenlänge 3,8-4,4 m; in Iasos: Tomasello (Anm. 33) 150 ff.; Hierapolis: E. Schneider Equini, La necropoli di Hierapolis di Frigia (1972) 113 f. Taf. 17a: 3,5 × 3,7 m; in Pisidien: A. Schulz, Ariassos, in: E. Schwertheim, Forschungen in Pisidien, Asia Minor Studien 6 (1992) 29 ff. mit Abb. 1 (Plan).

40 Berns (2003) 136 mit Bibliographie. Rekonstruktionen werden aufgrund der »typologischen Vielfalt an Grabbauten und der beliebigen Kombinierbarkeit« erschwert: Berns (2003) 74 mit Bezug auf die Rekonstruktion der Grabbauten, deren Platten in der Marienkirche von Ephesos verbaut wurden.

${ }^{41}$ Die Problematik der 'Akkulturationsprozesse' wurde erst kürzlich in einem Kongress in Wien diskutiert, s. M. Meyer (Hrsg.), Neue Zeiten - Neue Sitten. Zu Rezeption und Integration römischen und italischen Kulturguts in Kleinasien, WForsch 12 (2007); s. auch M. Meyer, Neue Zeiten - Neue Sitten. Kolloquium Universität Wien 2005, Forum Archaeologiae 35/VI/2005 (http://farch. net), welche die Prozesse in Reflexion auf »Reaktionen auf Kulturkontakte« definiert. Zu den Italikern in Ephesos s. zuletzt: F. Kirbihler, Die Italiker in Kleinasien, mit besonderer Berücksichtigung von Ephesos (133 v. Chr. - 1. Jh. n. Chr.), in: Meyer a. O. 19 ff. 
Während die Mauertechnik des Grabhauses 3 auf eine Entstehung kaum vor dem 1. Jahrhundert v. Chr. hinweist, ergibt sich eine stratigraphische Bestätigung dieser Datierung nur indirekt aus den Befunden zweier ungestörter Gräber (Grab 1 und 2, s. Abb. 3), die direkt an die Nordostseite des Grabhauses 3 angesetzt wurden. Die beiden Bestattungen wurden in eine $1 \times 2 \mathrm{~m}$ große und $0,80 \mathrm{~m}$ tiefe Grube (Stratigraphische Einheit [im Folgenden: SE] 101a/05) gelegt und an der Oberseite mit Steinen verschlossen. Grab 1 wurde mit acht unbearbeiteten Kalksteinen abgedeckt ${ }^{42}$, deren Oberkante ${ }^{43}$ mit der erhaltenen Mauerkrone des Grabhauses 3 abschloss (s. Abb. 3. 5). Die Steine lagen auf einem braunen, sandig-lehmigen Stratum (SE 103/05), das die eigentliche Grablege abschloss ${ }^{44}$. Die Grablege bestand aus zwei Gefäßen ${ }^{45}$, die in einem lehmigsandigen Stratum (SE 105/05) eingebettet waren. Die Gefäße beinhalteten verbrannte menschliche sowie nicht näher spezifizierbare tierische Knochenreste ${ }^{46}$. Die beiden gebrauchskeramischen Gefäße, die in die Zeit um 100 v. Chr. datieren ${ }^{47}$, waren übereinander gestellt; das untere ist nur zur Hälfte erhalten.

In einem Abstand von 1,50 m nach Südwesten befindet sich Grab 2. In ihm wurde der Kiefer eines 3-4 Jahre alten Kindes gefunden, deponiert in einem Gefäß, welches nach Auskunft S. Ladstätters in die Zeit um 100 v. Chr. datiert. Mit Ausnahme des Kiefers, der keine Verbrennungsspuren aufweist, haben sich keine Knochenreste gefunden. Überraschend war die Abdeckung des Grabes: Die konvex gewölbte Steinplatte mit Resten eines kleinen Säulenfußes erwies sich als Rückseite eines bemalten Grabreliefs ${ }^{48}$, das hier - in Zweitverwendung - als Deckstein des Grabes 2 diente ${ }^{49}$. Die Südostseite des Grabes wurde mit einer kleinen, horizontal stehenden Marmorplatte verschlossen ${ }^{50}$. Diese Platte weist an den Schmalseiten jeweils ein Halterungsloch und an der Unterseite einen Dübel auf; auf der Oberseite befinden sich vier großteils abgebrochene 'Eckakrotere'. Offenbar handelt es sich um eine Platte, die von einer nahe gelegenen Grabanlage stammt und hier als Seitenwand des Grabes verwendet wurde. Da aufgrund der Lage des Reliefs direkt an der Südostkante des Grabhauses 3 auszuschließen ist, dass sich das Relief in Sturzlage befindet, muss es sich wie bei der Marmorplatte um eine bewusste Deponierung handeln, in der Absicht, das Grab des Kindes zu verschließen ${ }^{51}$.

Beide Grablegen wurden in einer Grube (SE 101a/05) deponiert, die mit lehmig-sandigem Material (SE 103/05, 105/05) aufgefüllt wurde. Allem Anschein stammen beide Gräber aus derselben Zeit. Aufgrund des direkten Anschlusses an die Südostmauer des Grabhauses 3 und auch wegen fehlender Hinweise auf eine Störung ist davon auszugehen, dass sie nach der Errichtung des Grabhauses 3 angelegt wurden. Die Datierung der Keramik entspricht der Entstehungszeit des Reliefs ${ }^{52}$, weshalb davon auszugehen ist, dass ihre Anlage in die Zeit kurz nach 100 v. Chr. fällt. Da zu diesem Zeitpunkt das Grabhaus 3 bereits errichtet gewesen sein musste, kann dieses wohl gegen den Beginn des 1. Jahrhunderts v. Chr. datiert werden.

Im Zusammenhang mit der Nekropole am Ostabhang des Panayır Dağ stellt sich Grabhaus 3 in die Reihe anderer bereits bekannter Grabhäuser ${ }^{53}$ und dokumentiert mit dem Grabrelief die Nutzung dieser Nekropole in späthellenistischer Zeit. Die beiden Grabhäuser 1 und 2 nördlich des Grabhauses 3 gehören zu einem Grabbezirk, der an die Damianosstoa anschließt und aufgrund der parallelen Grabbauten im Bereich der Stoa wohl gegen Ende des 2./Anfang des 3. Jahrhunderts n. Chr. datiert.

A. Sokolicek

$420,50 \times 0,70 \mathrm{~m}$.

$4317,76 \mathrm{~m}$ absolute $\mathrm{H}$.

${ }^{44}$ UK 17,00 m (absolute H).

${ }^{45}$ Inv. 3/01-05 und 3/03-05.

${ }^{46}$ Freundliche Auskunft K. Grossschmidt und F. Kanz, die für die anthropologische Bestimmung aller in den Gräbern gefundenen Knochen verantwortlich zeichnen.

${ }^{47}$ Freundliche Auskunft S. Ladstätter.

${ }^{48}$ Fundnr. 3/04.

${ }^{49}$ Zum Grabrelief s. u. den Beitrag von J. Auinger.

${ }^{50}$ Fundnr. 3/05.

${ }^{51}$ Schließungen einfacher Gräber mit Deckeln sind auch aus anderen hellenistischen Nekropolen bekannt, s. Schmidt (1991) 4; zu Bestattungen in einfachen Gneisplatten s. v. Moock (Anm. 38) 375 mit Anm. 7 und Taf. 90, 2.

${ }^{52}$ s. u. Beitrag J. Auinger.

${ }^{53}$ Knibbe - Langmann (1993); Thür (1999). 


\section{Das Grabrelief $^{54}$}

Das beinahe quadratische, konkav gewölbte Relief ${ }^{55}$ überraschte bei seiner Auffindung durch partielle Farbreste $^{56}$, die an der nur noch an wenigen Stellen original erhaltenen Oberfläche anhaften. Die Vorderseite (Abb. 8) wird von einem langrechteckigen Reliefbild bestimmt. Der obere Rand ist in einem unregelmäßigen Bruchverlauf horizontal abgeschlagen, sodass die ursprüngliche Höhe des Reliefbildes und dessen Abschlussgestaltung nicht mehr ermittelt werden können ${ }^{57}$. Auf einer mit dem Spitzmeißel grob behauenen Standleiste, deren oberes Drittel geglättet ist und zu beiden Seiten leicht ansteigt, entwickelt sich ein flaches Relief. Fünf Personen sind in eindeutigen Bezügen zueinander dargestellt. Die zentrale Figur - ein nach links gewendeter Jüngling - befindet sich in der Mitte der Komposition. Er ist durch Handschlag mit einer vor ihm sitzenden Frau verbunden. Dieser sitzt eine zweite Frau axialsymmetrisch gegenüber, wobei beide Frauen von je einer

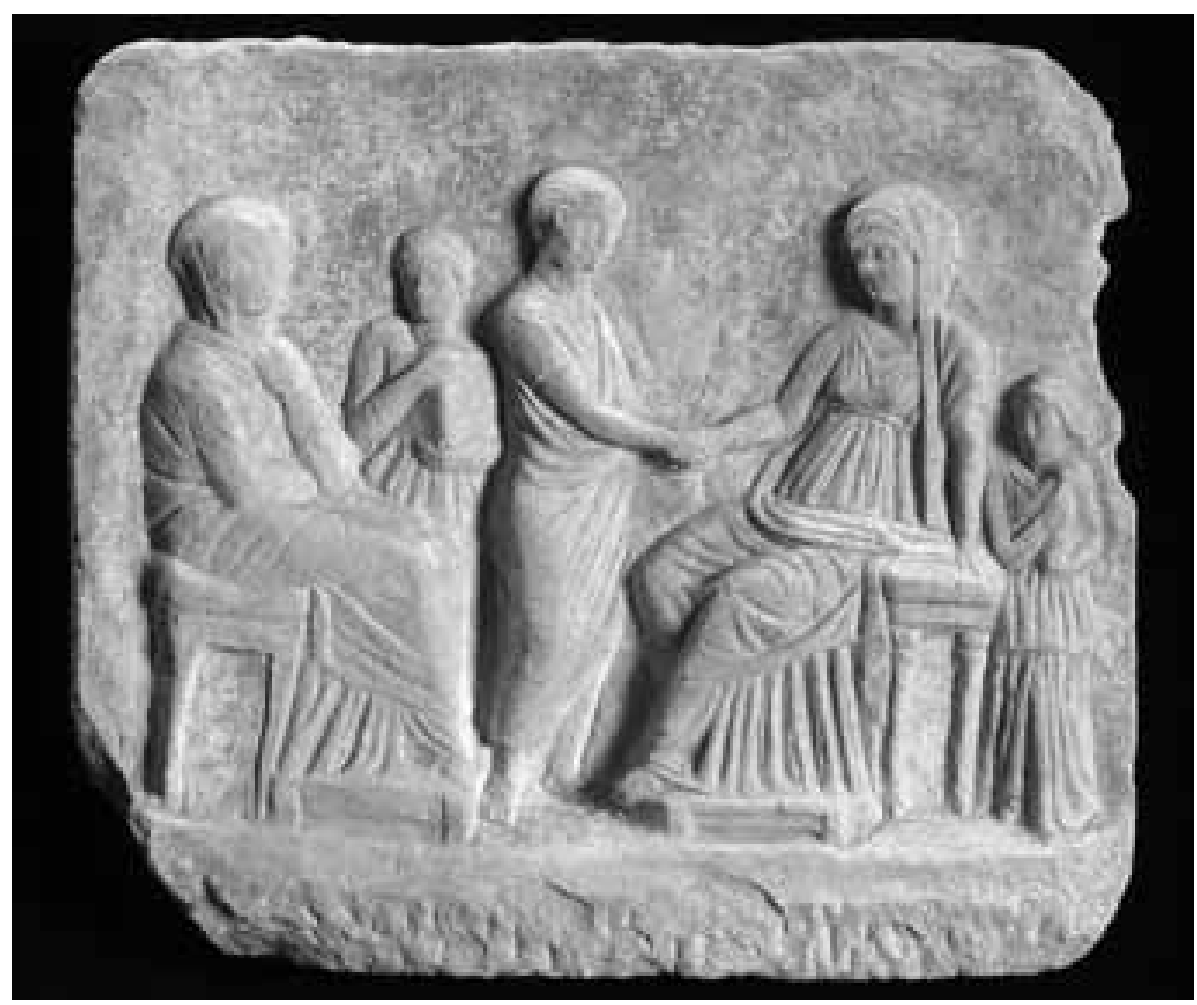

8 Grabrelief (Vorderseite)

Dienerin begleitet werden. Dem Relief fehlt jegliche Raumangabe durch Gestaltung des Hintergrundes; dieser war - was die Farbreste noch erkennen ließen - in einem einheitlich aufgetragenem Blau gehalten ${ }^{58}$. $\mathrm{Zu}$ beiden Seiten der Darstellung ist als äußere Begrenzung je ein vertikaler Streifen von der Farbfassung ausgespart. Eine Inschrift ${ }^{59}$, die eine genauere Interpretation der Szene ermöglichen würde, ist weder auf der Standleiste noch oberhalb der Figuren erhalten. Möglicherweise ist das Thema des Reliefs auf die am linken Bildrand sitzende Frau bezogen: Sie ist deutlich von der rechten Personengruppe abgesetzt und darüber hinaus als Einzige - abgesehen von

${ }^{54}$ Dem Grabungsleiter F. Krinzinger und dem ausführenden Archäologen vor Ort A. Sokolicek sei herzlichst für die Einladung, das während der Grabungen gefundene Relief zu publizieren, gedankt.

${ }^{55}$ Eine naturwissenschaftliche Marmoruntersuchung mithilfe der Isotopenanalyse konnte in der zur Verfügung stehenden Zeit nicht erfolgen. Mithilfe einer makroskopischen Untersuchung durch die Verf. konnte ein grobkörniger, hellgrauer Marmor festgestellt werden. Farbe und Körnung lassen an die Provenienz aus einem lokalen ephesischen Steinbruch denken, sodass Ephesos als Herstellungsort nicht auszuschließen ist. - Maße: H 0,391 m; L 0,446 m; T (max.) 0,07 m; Standleiste H (max.) 0,056 m; Relief-T (max.) $0,036 \mathrm{~m}$.

${ }^{56}$ Das Relief war bei seiner Auffindung stark versintert. Es wurde durch K. Herold (ÖAI) einer Laserreinigung unterzogen. Der Verlust von Farbe konnte durch die starke Versinterung der Oberfläche nicht verhindert werden.

${ }^{57}$ Es ist anzunehmen, dass der Abschluss unmittelbar nach der Bruchkante folgt. Das Reliefbild ist zu beiden Seiten weder durch Leisten, Pfeiler oder Säulen begrenzt, sodass der obere Abschluss ähnlich den seitlichen zu rekonstruieren ist, nämlich als kaum merkbarer, erhöhter Rand. Vgl. etwa ein Totenmahlrelief aus dem Hanghaus 2 in Ephesos, zuletzt: E. Rathmayr, Skulpturen, in: H. Thür (Hrsg.), Hanghaus 2 in Ephesos. Die Wohneinheit 4. Baubefund, Ausstattung, Funde, FiE VIII 6 (2005) 208 ff. 227 S4 Taf. 138, 3.

${ }^{58}$ Zur Farbigkeit zuletzt: V. Brinkmann, Die blauen Augen der Perser. Die farbige Skulptur der Alexanderzeit und des Hellenismus, in: ders. - R. Wünsche (Hrsg.), Bunte Götter. Die Farbigkeit antiker Skulptur. Eine Ausstellung der Staatlichen Antikensammlung und Glyptothek München, 16. Dezember 2003 - 29. Februar 2004, 15. Juni - 5. September 2004 (2004) 166 ff.

${ }^{59} \mathrm{Zu}$ Grabepigrammen s. die Sammlung in der Anth. Gr. 7; W. Peek, Griechische Grabgedichte (1960). 
den Dienerinnen - aus der Szenerie herausgedreht ${ }^{60}$. In welchem Verhältnis sie zu dem Jüngling und der anderen Sitzenden steht, zu Personen, die aus dem familiären Umfeld der Hauptperson stammen dürften, ist im Bereich des Spekulativen anzusiedeln.

Der Marmorblock, aus dem das Relief hergestellt wurde, hatte ursprünglich eine andere Verwendung. Die konvex gewölbte Rückseite (Abb. 9), auf der mittig in der oberen Hälfte eine kreisrunde Erhöhung erhalten ist, gibt Aufschluss über seine Primärverwendung: Die Erhöhung könnte auf die Existenz einer runden Basis oder eines SäulenfuBes hinweisen. Der Block ist

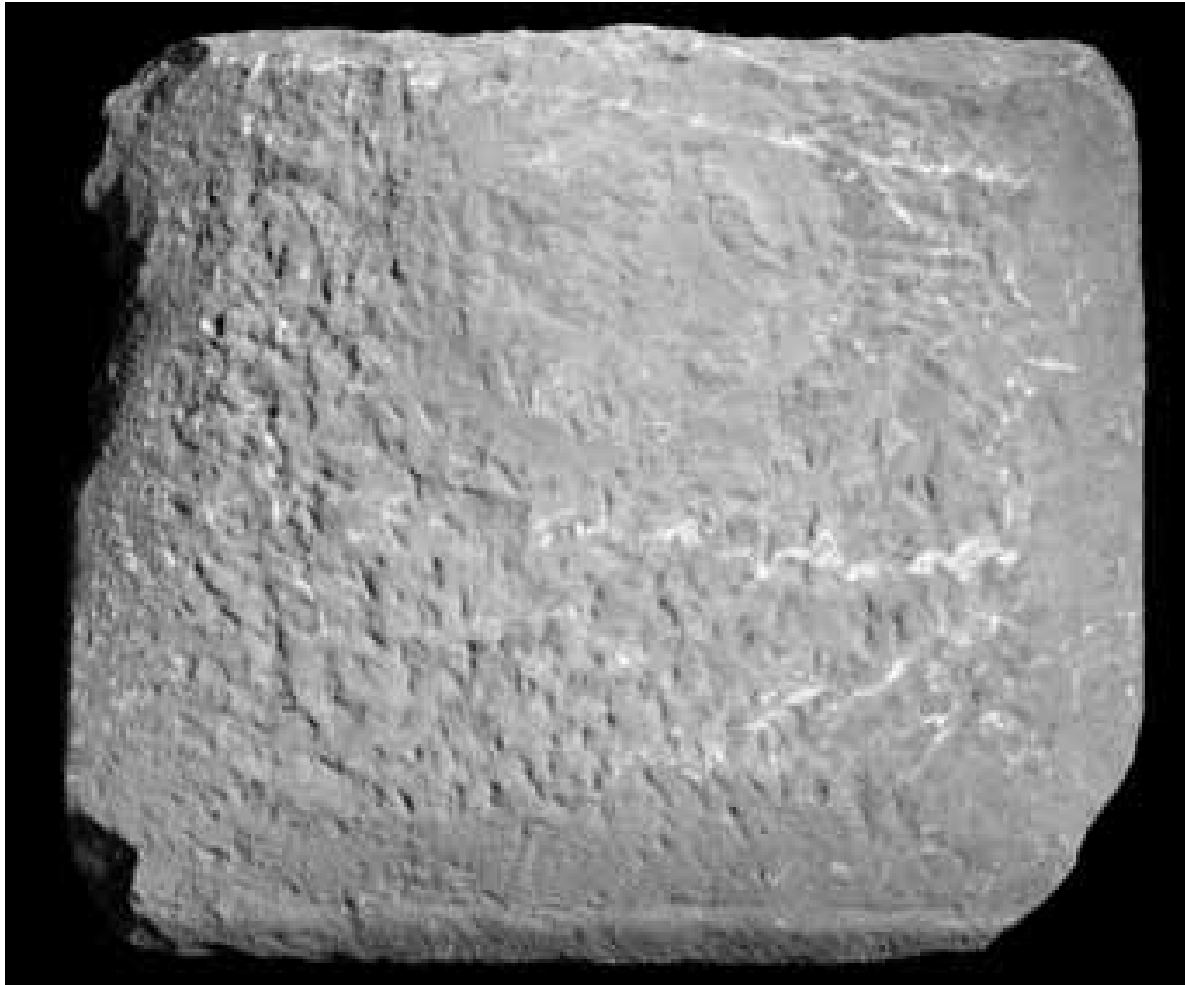

9 Grabrelief (Rückseite) somit als Teil einer Stylobatplatte anzusprechen, deren Dimensionen sowohl durch den unteren als auch die seitlichen, anscheinend originalen Ränder festgelegt werden.

Die Platte weist keinerlei Zurichtungen für eine Befestigung auf, seien es Klammer- oder Dübellöcher oder einen unterhalb des Reliefs befindlichen mittigen Zapfen für die Einlassung in eine Stele ${ }^{61}$. Wenn das Relief - wie anzunehmen - für den Besucher des Grabes sichtbar versetzt war, muss wohl eine Anbringung in einem architektonischen Verband angenommen werden ${ }^{62}$. Das nördlich angrenzende Grabhaus 3 ist bis zu seinem Fundament beraubt und kann aufgrund der Keramik aus den angrenzenden Gräbern lediglich relativ älter datiert werden ${ }^{63}$. Vorstellbar ist auch eine Befestigung durch Haken an der Ober- und Unterseite des Reliefs ${ }^{64}$. Handelt es sich um ein 'verschlepptes' Relief ${ }^{65}$, d. h., dass es ursprünglich in einen anderen Kontext zu stellen ist, so ist auch eine Anbringung durch die Einlassung in eine Grabstele mithilfe einer dafür vorgesehen Vertiefung in Erwägung zu ziehen ${ }^{66}$. Dennoch scheint ein intentioneller Zusammenhang zwischen dem Relief und der Grablege des Kindes zu bestehen ${ }^{67}$.

\footnotetext{
${ }^{60}$ Zur besonderen Hervorhebung der dargestellten Toten auf Marmorlekythen vgl. die Ausführungen von B. Schmaltz, Untersuchungen zu den attischen Marmorlekythen (1970) $95 \mathrm{ff}$.

${ }^{61} \mathrm{Zu}$ technischen Aspekten in Bezug auf die Anbringung von Grabreliefs s. Pfanner (1989) 165 ff. bes. 183 ff. Bei einigen der samischen Totenmahlreliefs finden sich ebenfalls keine Vorrichtungen von Dübel- oder Klammerlöchern, sie werden in den Mauerverband vertieft rekonstruiert (s. dazu Fabricius [1999] 136 f.).

${ }^{62} \mathrm{Zu}$ Einlassungen von Grabreliefs in Mauerverbände in Ephesos s. die sog. Grabmauern mit Gladiatorengrabsteinen aus dem 2. Jh. n. Chr., zuletzt bei Pietsch (1999) 455 ff. bes. 459. Zur Verdeutlichung der möglichen Anbringung sowohl in einer Stele als auch in einer Mauer s. Pfanner (1989) 184 Abb. 8 C.a. Dadurch würden sich die seitlichen Ränder ohne Farbe erklären und der untere, grob gepickte Abschluss.

${ }^{63}$ S. o. die Ausführungen von A. Sokolicek.

${ }^{64}$ Pfanner (1989) 184 Abb. 8 C.c.d.

${ }^{65}$ Zur Wiederverwendung von Grabreliefs s. P. M. Fraser - T. Rönne, Boeotian and West Greek Tombstones (1957) 161 ff.; Pfuhl - Möbius I 75 f.

${ }^{66}$ Pfanner (1989) 184 Abb. 8 A.c.

${ }_{67} \mathrm{Zu}$ dem Kontextproblem hellenistischer Grabreliefs äußerte sich Pfanner (1989) 183: »Es ist sicherlich eines der merkwürdigsten Phänomene in der Forschungsgeschichte hellenistischer Grabreliefs, daß wir zwar eine in die Tausend gehende Anzahl von Stü-
} 


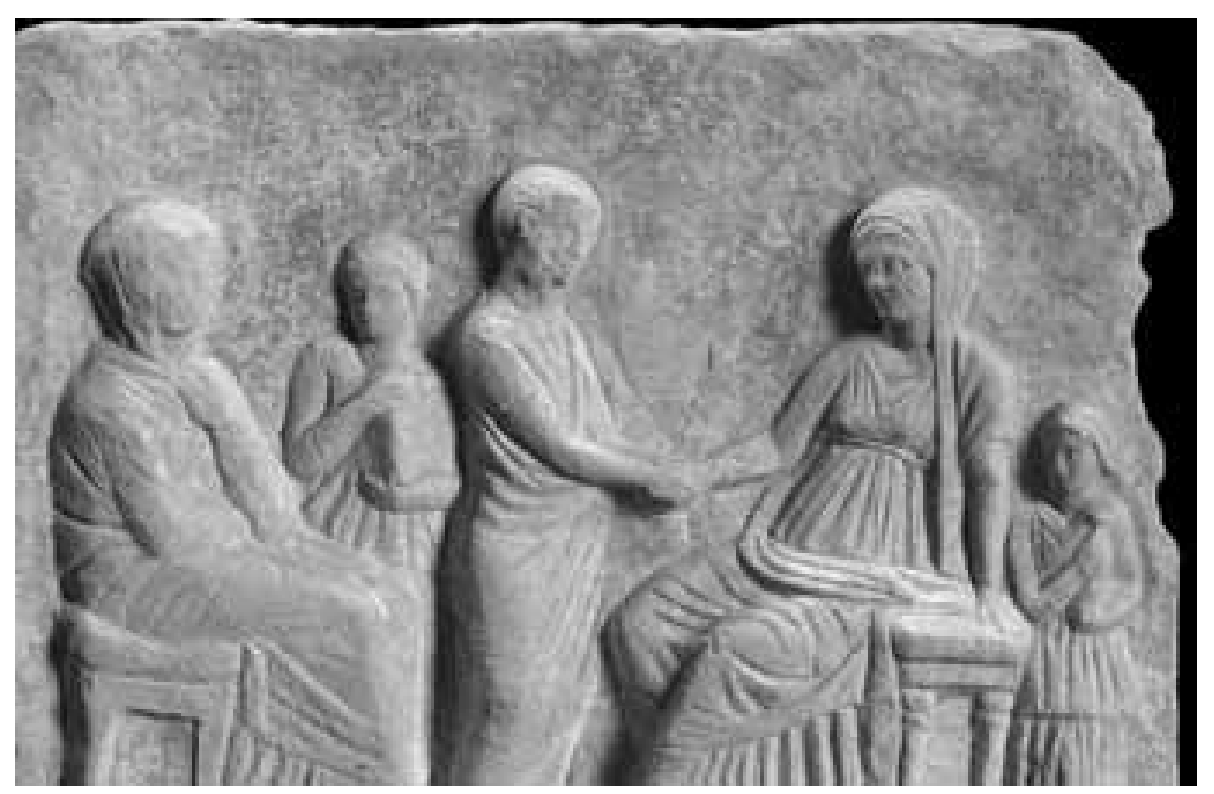

10 Grabrelief (Detail)

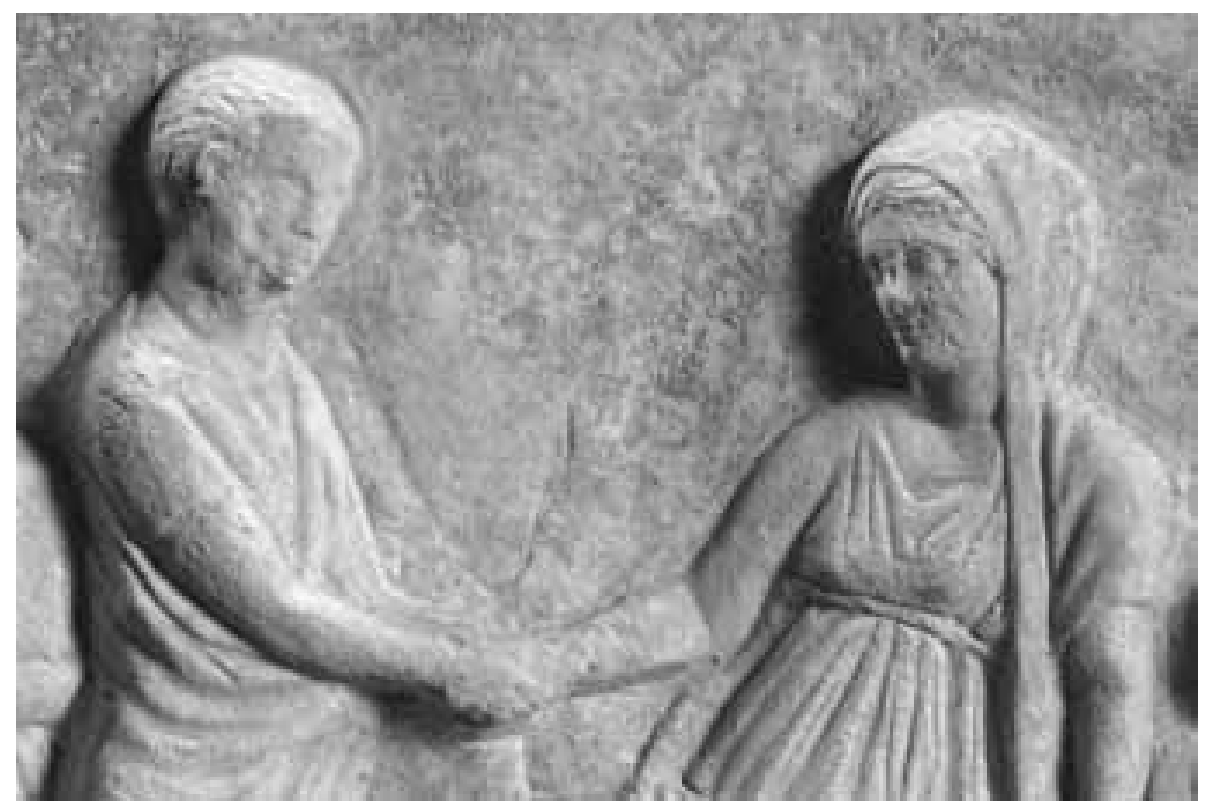

11 Grabrelief (Detail)
Im Mittelpunkt der Darstellung steht der nach links gewendete Jüngling (Abb. 8. 10-12), der das linke Bein in einer offenen Schrittstellung belastet. Der Oberkörper ist aus der Seitenansicht leicht herausgedreht. Dieser Bewegung folgt - jedoch wesentlich stärker - der Unterkörper mit der Hüftpartie, sodass der rechte Unterschenkel mit dem Knie frontal erscheint. Die Füße sind nackt; Sandalenriemen waren vielleicht in Farbe angegeben. Gekleidet ist der Jüngling mit einem Himation, welches die rechte Schulter und den Oberkörper frei lässt und so den darunterliegenden Chiton, der bis zur Mitte des Oberarmes reicht, zeigt ${ }^{68}$. Das Himation führt von der Spielbeinhüfte schräg über den Oberkörper zur linken Schulter; das über der linken Schulter anzunehmende Gewandende ist nicht dargestellt ${ }^{69}$. Das Himation endet oberhalb des rechten Fußknöchels, sein Saum führt steil zum Standbeinknie empor, sodass der Unterschenkel unbedeckt bleibt. Eng legt es sich um das Spielbein, wodurch sich der Kontur des Oberschenkels deutlich abzeichnet.

Zwischen den Beinen entwickeln sich steil geführte Spannfalten, oberhalb der Spielbeinhüfte staut sich das Himation in weitgezogenen Bogenfalten. Die Gewandwiedergabe ist allgemein schematisch ausgeführt: Die Falten folgen weder den Körperformen noch unterstreichen sie diese, sondern sind ohne Variationen parallel nebeneinanderliegend in die Gewandoberflächen eingetieft. Der halsferne Chiton ist in gleicher Weise durch wenige Falten charakterisiert.

Den Kopf hat der Jüngling (Abb. 11. 12) in Dreiviertelansicht leicht gesenkt und zu der Sitzenden, mit welcher er im Handschlag verbunden ist, gedreht. Seine kappenartige Kurzhaarfrisur, die die Stirnecken und

cken besitzen, aber so gut wie bei keinem den Kontext kennen.«

${ }^{68}$ Die Form des Jünglingsmantels und die nackten Füße finden sich z. B. bei einem Relief aus Delos wieder: Couilloud (1974) 79 Nr. 49 Taf. 9 (Ende 2. /Anfang 1. Jh. v. Chr.).

${ }^{69} \mathrm{Zu}$ den Typen der Himationträger im Hellenismus s. v. a. mit älterer Bibliographie A. Lewerentz, Stehende männliche Gewandstatuen im Hellenismus. Ein Beitrag zur Stilgeschichte und Ikonologie hellenistischer Plastik (1993). 
das Ohr frei lässt, rahmt in einem regelmäßigen Bogen die hohe Stirn. Diese Frisur, die sich der Kopfform eng anpasst, findet sich bei einigen Porträtköpfen aus Delos wieder ${ }^{70}$, die »short-cropped hair, and a hard, objective, realistic-looking style $\ll^{71}$ aufweisen. Die Haarstruktur ist durch regelmäßig gesetzte Pickungen erzeugt. Das Gesichtsfeld des Jünglings ist stark abgerieben, weswegen seine Physiognomie undeutlich und wenig prägnant erscheint. Aus dem dreieckigen Gesichtsfeld, das durch ein kleines Kinn abgeschlossen wird, tritt die kräftige Nase dominant hervor. Der Mund wird durch volle Lippen charakterisiert. Die gleichmäßig geschwungenen Brauenbögen zeichnen sich deutlich ab. Der Jüngling überragt im Stehen die beiden Sitzenden minimal.

Die junge Frau (Abb. 8. 10-11), der sich der Jüngling zuwendet, hat sich auf einem nur von der Seite sichtbaren Diphros mit Polsterauflage niedergelassen. Die Beine des Diphros sind konisch mit einem Einzug am oberen Ende, an dem als Verbindung zur Sitzfläche kugelförmige Elemente aufliegen $^{72}$. Die Frau sitzt mit leicht gespreizten Beinen, wobei sie das linke Bein weit nach vorn setzt, sodass die Fußspitze über den Rand des Schemels ragt. Das rechte Bein ist angewinkelt und ab der Mitte des Unterschenkels vom linken Bein verdeckt. Die Füße ruhen auf einem ebenso in strenger Seitenansicht dargestellten, einfach profilierten Fußschemel. Der Oberkörper ist stark aus der Seitenansicht he-

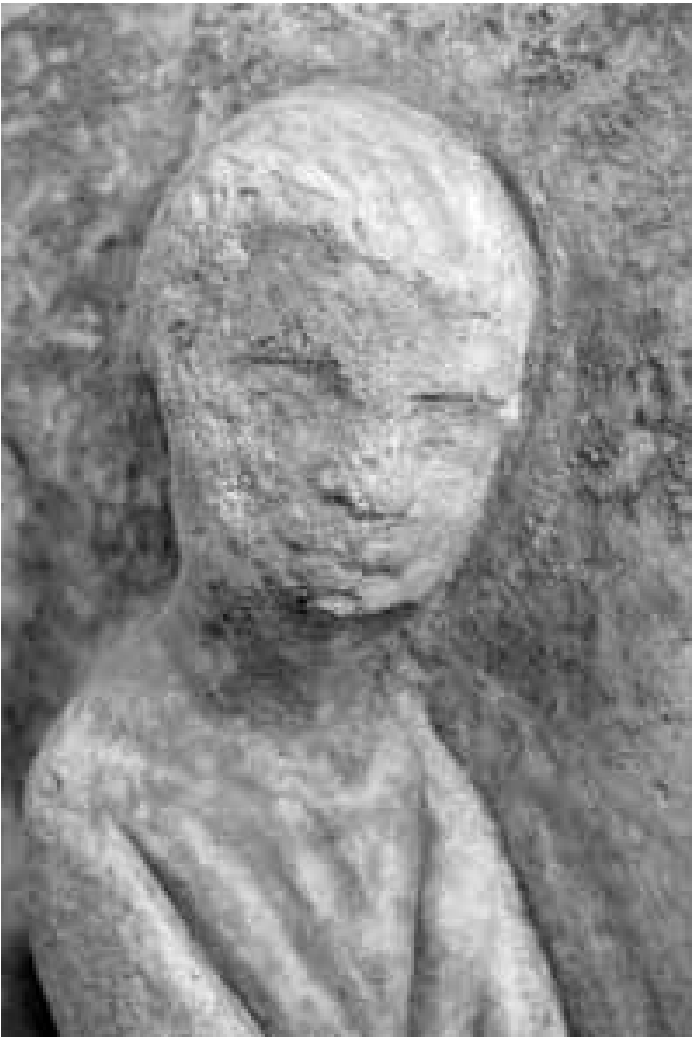

12 Grabrelief (Detail) rausgedreht, sodass er dem Betrachter nahezu frontal zugewendet ist, hingegen ist die Beinpartie etwas stärker zur Seite gerichtet. Mit der linken Hand stützt sich die Frau auf dem hinteren Teil des Stuhles $a^{73}$, die rechte Hand reicht sie dem Jüngling. Sie trägt einen unter der Brust gegürteten, vielfach gefältelten Chiton und darüber einen Mantel, der blasslila gefasst war ${ }^{74}$. Er wird über das leicht gesenkte Haupt geführt, fällt über die linke Schulter und bedeckt den Oberarm bis knapp zum Ellbogen. An der rechten Flanke zieht er in Hüfthöhe zur Vorderseite, lässt den Oberkörper aber unbedeckt. Der Mantel staut sich im Schoß zu einem deutlich abgesetzten Bausch, dessen Ende in einer langgezogenen Schlaufe auf dem Polster liegt und dessen Zipfel in einer schmalen Stoffbahn neben dem vorderen Stuhlbein herabgleitet. Am Unterkörper reicht der Mantel bis unterhalb der Kniepartie. Auch bei der Gewandung der Sitzenden werden die Falten in einem starren Schematismus über die Körperformen gelegt. Die weiche, sich zum Kinn hin stark verjüngende Gesichtsform mit einem kleinen, hervortretenden Kinn, die durch ihre Größe hervorgehobenen Augenwölbungen und der Mund mit vollen Lippen charakterisieren das Antlitz der

\footnotetext{
${ }^{70}$ Hier seien vor allem die Köpfe Delos A 2912 (= A. Stewart, Attika. Studies in Athenian Sculpture of the Hellenistic Age [1979] Abb. 18c) und A 4186 (= Stewart a. O. Abb. 19d; R. R. R. Smith, Hellenistic Sculpture [1991] 256 Taf. 72, 1 - mit einer Datierung um 100 v. Chr.) genannt. Zum Problem der Chronologie vgl. Stewart a. O. 65 ff. bes. 68 Tab. 1. Ferner sei auf das Kopffragment aus Delos bei C. Michalowski, Les Portraits hellénistiques et romains, Délos XIII (1932) 35 ff. Taf. 26 (»Maske eines Mannes«, Datierung Ende/Anfang 1. Jh. v. Chr.) verwiesen. - Zu Porträtköpfen in hellenistischer Zeit s. allgemein: G. Hafner, Späthellenistische Bildnisplastik (1954); E. Buschor, Das hellenistische Bildnis² (1971); R. R. R. Smith - G. M. A. Richter, The Portraits of the Greeks (1984). Zu Datierungsschwierigkeiten von Privatporträts im Hellenismus s. K. Fittschen, Griechische Porträts (1988) 22.

${ }^{71}$ Smith (Anm. 70) 256.

72 s. allgemein Pfuhl - Möbius II 361 ff. Nach G. M. A. Richter, The Furniture of the Greeks, Etruscans and Romans (1966) $38 \mathrm{ff}$. bes. 39 f. handelt es sich bei dem Diphros um Typ I, jenen Typ, bei dem ein kugelförmiges Element zwischen Sitzfläche und Stuhlbeinen eingefügt ist.

${ }^{73}$ Diese Haltung findet sich bereits auf attischen Grabreliefs wieder, s. z. B. C. W. Clairmont, Classical Attic Tombstones (1993) Kat. Nr. 3.446; 3.490.

${ }^{74}$ Die Farbreste befinden sich am Mantel über dem Kopf und an dem über die linke Schulter gleitenden Mantelteil.
} 
jungen Frau (Abb. 11) ${ }^{75}$. Das über der Stirnmitte gescheitelte Haar streicht in einzelnen geschwungenen Lockensträhnen zu beiden Seiten des Gesichts. Am Hinterkopf zeichnet sich unter dem Mantel ein hochsitzender Haarknoten ab, eine Frisur, die bis zur Mitte des 1. Jahrhunderts v. Chr. beliebt war ${ }^{76}$.

Hinter dem Stuhl steht frontal und ihr rechtes Bein belastend eine als kleines Mädchen dargestellte Dienerin (Abb. 8. 10), die der Frau bis zur Brust reicht. Sie ist mit einem Peplos bekleidet, dessen Überfall bis zur Mitte der Oberschenkel herabgleitet. Die Schuhspitzen ragen unter dem Gewand hervor. Der Kopf ist leicht gesenkt und zur rechten Seite gewendet. Die haubenartige Frisur des über der Stirnmitte gescheitelten voluminösen Haares rahmt das Gesicht und ist unterhalb der Ohren nach hinten genommen. Eine auffallende Übereinstimmung der Frisur, die ab dem späten 4. Jahrhundert während des gesamten Hellenismus beliebt war, zeigt ein weiblicher Porträtkopf von Kos, der um die Mitte des 2. Jahrhunderts v. Chr. datiert wird ${ }^{77}$. Das Gesicht der Dienerin ist - ohne charakterisierende Wiedergabe der Physiognomie - durch sphärische Formen bestimmt. In den Händen hält sie ein Alabastron ${ }^{78}$ : Mit der linken Hand unterfängt sie das Gefäß, mit der rechten drückt sie es an die linke Oberkörperseite.

Die zweite Sitzende ist im Typus der Pudicitia ${ }^{79}$ mit bedecktem Haupt dargestellt (Abb. 8. 10). Beide Arme sind von dem Mantel verhüllt und unter diesem verborgen. Der rechte Arm ist angewinkelt in den Schoß gelegt, der linke Arm stützt sich auf den Oberschenkel und ist vertikal zur Mitte des Halses geführt ${ }^{80}$, wo die Hand den Mantel rafft. Die Frau sitzt seitlich mit leicht gerundetem Rücken ebenfalls auf einem Diphros mit Polsterauflage, dessen Beine jedoch ohne Profilierung einfacher gestaltet sind ${ }^{81}$. Die Füße ruhen auf einem unprofilierten Fußschemel ${ }^{82}$. Der Mantel lässt die Unterschenkelpartie frei, sodass der gefältelte Chiton, der in vertikalen parallelen Falten fällt, sichtbar wird. Die in die Stoffoberfläche des Mantels eingetieften Falten folgen schematisch den Körperformen. Das Haupt ist so stark beschädigt, dass keine Aussagen zu Physiognomie oder Frisur möglich sind.

Auch diese Frau wird von einer mit einem Peplos bekleideten Dienerin begleitet (Abb. 8. 10), die im Stehen etwas kleiner als ihre Herrin ist. Das Mädchen steht frontal neben ihr, sodass ihr Unterkörper von der Beinpartie der Herrin verdeckt ist. Die Dienerin hält einen Gegenstand in den Händen, der infolge des schlechten Erhaltungszustandes als Alabastron, wahrscheinlicher aber als Kästchen mit zwei sichtbaren kleinen Füßen zu interpretieren ist. Ihre Aufmerksamkeit gilt der Kopfhaltung und ihrer Blickrichtung nach zu urteilen der Sitzenden. Ihr Haar ist über der Stirnmitte gescheitelt und umspielt zu beiden Seiten das runde Gesicht, dahinter ist das Kalottenhaar deutlich flacher abgesetzt. Die Physiognomie weist keine charakteristischen Züge auf.

${ }^{75}$ Vgl. den Kopf der Baebia-Statue aus Magnesia am Mäander, s. Kabus-Preisshofen (1989) 154 f. 156. 245 ff. Kat. 56 Taf. 63 , $1-3 ; 64,1-3$.

76 s. Pfanner (1989) 171 mit Anm. 5.

77 s. Kabus-Preisshofen (1989) 172 ff. 318 ff. Kat. 114 Taf. 78, 1. 2.

${ }^{78} \mathrm{Zu}$ den vermittelten weiblichen Idealen aufgrund der von Dienerinnen in den Händen gehaltenen Gegenständen s. Pfanner (1989) $176 \mathrm{f}$.

${ }^{79}$ Zur Typologie der stehenden Frauen im Pudicitia-Typus s. zuletzt ausführlich mit Forschungsgeschichte und älterer Bibliographie J. C. Eule, Hellenistische Bürgerinnen aus Kleinasien. Weibliche Gewandstatuen in ihrem antiken Kontext (2001) 15 ff.; grundlegend: R. Horn, Stehende weibliche Gewandstatuen in der hellenistischen Plastik, 2. Ergh. RM (1931) 21 f. 65 ff. 92 f.; ders., Hellenistische Bildwerke auf Samos, Samos XII (1972) 21 f.; D. Pinkwart, Weibliche Gewandstatuen aus Magnesia, AntPl 12 (1973) 149 ff.; A. Linfert, Kunstzentren hellenistischer Zeit. Studien an weiblichen Gewandfiguren (1976) 147 ff.; Pfuhl - Möbius I 63 f. Zum Versuch, die sitzenden Pudicitia-Typen zu differenzieren s. Cremer (1991) 81 ff. Gegen diese Einteilung spricht sich Fabricius (1999) 284 Anm. 36 zu Recht aus, da Cremer »häufig spiegelbildlich vertauschte Armhaltungen unter die jeweiligen Typen subsumiert«. Ferner s. L. Koch, Weibliche Sitzstatuen der Klassik und des Hellenismus und ihre kaiserzeitliche Rezeption. Die bekleideten Figuren (1994) 123 ff., die aber nur zwei Sitzende unterscheidet, Koch a. O. 129 ff. (Sitzstatuen im Typus der Saufeia und der Statue Braccio-Nuovo). Frauen im Pudicitia-Typus sind nicht primär in einem Trauergestus dargestellt, sondern nach P. Zanker, Brüche im Bürgerbild? Zur bürgerlichen Selbstdarstellung in den hellenistischen Städten, in: Stadtbild und Bürgerbild im Hellenismus. Kolloquium München 24. bis 26. Juni 1993, Vestigia 47 (1995) 263 handelt es sich um eine »eingeübte Haltung, weibliche Zurückhaltung und Scheu«, die »ins Bild gesetzt« wird.

80 Demnach wäre sie im Pudicitia-Typus der Baebia dargestellt; vgl. o. Anm. 76.

${ }^{81}$ s. allgemein Pfuhl - Möbius II 361 ff. Nach Richter (Anm. 72 ) 38 ff. bes. 42 handelt es sich bei dem Diphros um Typ V mit einfachen, geraden Füßen.

82 s. allgemein Richter (Anm. 72 ) 49 ff.; Pfuhl - Möbius II 363. 
Das neugefundene Grabrelief ist aufgrund stilistischer und ikonographischer Kriterien, die auf hellenistische Grabreliefs zutreffen ${ }^{83}$, in die Zeit des Hellenismus zu datieren.

In Ephesos und seiner Umgebung wurden einige hellenistische Grabreliefs gefunden, allerdings ist der Kontext dieser Zufallsfunde nicht bekannt, zumal es keine systematischen Grabungen in den Nekropolen ${ }^{84}$ gab. Die Reliefs wurden in den nach typologischen Kriterien geordneten Corpus der ostgriechischen Grabreliefs von E. Pfuhl und H. Möbius aufgenommen ${ }^{85}$, die später gefundenen und unpublizierten Reliefs hat E. Atalay vorgestellt ${ }^{86}$; zu herausragenden Stücken existieren mehrere Einzelstudien ${ }^{87}$. Mindestens zwei Reliefs konnten in der Nähe der Fundstelle unseres Reliefs - in diesem Gebiet geht E. Atalay ${ }^{88}$ auch von einer hellenistischen Nekropole aus - zu einem früheren Zeitpunkt sichergestellt werden ${ }^{89}$.

Ephesos ist nicht als bedeutendes Produktionszentrum hellenistischer Grabreliefs anzusprechen ${ }^{90}$, es weist $» k e i n e$ eigenständige Tradition im Sinne einer Kunstlandschaft ${ }^{91}$ auf. St. Schmidt listet in seiner monographischen Behandlung der hellenistischen Grabreliefs Exemplare einer einheimischen ephesischen Werkstatt auf, die sich durch den Formcharakter der Beischriften und die unruhige Faltenwiedergabe der Gewänder auszeichnet ${ }^{92}$. Für diese Gruppe dienten smyrnäische Reliefs als Vorbilder ${ }^{93}$. Hier ist eine für die Forschung wichtige Werkstätte bekannt ${ }^{94}$, von der sich auch Stücke in Ephesos befinden ${ }^{95}$. In Smyrna werden die Verstorbenen und die Hinterbliebenen gerne frontal stehend als »vorbildliche Bürger « charakterisiert ${ }^{96}$. Ferner sind unter dem ephesischen Material auch eindeutig delische Grabreliefs zu finden ${ }^{97}$ : Die Grabreliefs von Delos $^{98}$ zeichnen sich durch ihre Vorliebe für klassische - somit traditionelle - Schemata aus ${ }^{99}$. Viele Reliefs haben Dexiosis-Szenen zwischen einer sitzenden und einer stehenden Person zum Inhalt ${ }^{100}$. Weitere eigenständige Gruppen von Grabreliefs finden sich vor allem auf den dem kleinasiatischen Festland benachbarten

\footnotetext{
83 s. zusammenfassend Schmidt (1991) 42.

${ }^{84}$ Zuletzt zu den Nekropolen von Ephesos: Pietsch (1999) 455 ff.

${ }^{85}$ Pfuhl - Möbius I. II.

${ }^{86}$ Atalay (1973) 231 ff.; Atalay (1988). Ferner Grabreliefs aus dem Kaystrostal: R. Meriç, Neue ostgriechische Grabreliefs aus Ionien und Lydien. Mit einem historisch-topographischen Exkurs, ÖJh 62, 1993, 57 ff.

87 Zuletzt zu jenen aus dem Hanghaus 2 in Ephesos: Rathmayr (Anm. 56) 208 ff. 227 S2-S4 Taf. 138, 1-3 (mit älterer Bibliographie).

${ }^{88}$ Atalay (1973) 232. Grundlegend für eine Beschäftigung mit den ephesischen Nekropolen s. Pietsch (1999).

${ }^{89}$ s. o. Anm. 9.

90 s. dazu die Ausführungen von Schmidt (1991) $20 \mathrm{ff}$.

${ }^{91}$ Schmidt (1991) 21.

92 Schmidt (1991) 21 mit Anm. 130.

${ }_{93}$ Schmidt (1991) 21.

${ }^{94}$ Zur typologischen Einheit der Grabstelen von Smyrna s. Schmidt (1991) 9 ff.

95 Schmidt (1991) 21 mit Anm. 128.

96 Bürgerliche Repräsentation auf Grabreliefs von Smyrna, vgl. P. Zanker, The Hellenistic Grave Stelai from Smyrna. Identity and Self-image in the Polis, in: Images and Ideologies. Self-definition in the Hellenistic world. Conference held at Berkeley, April 7-9, 1988 (1993) 212 ff.; ders. (Anm. 79) 251 ff.; s. dazu auch mit der Verbindung Grabepigramm und Bild: Schmidt (1991) 117
} ff.

${ }^{97}$ Schmidt (1991) 21 Anm. 129; zur Verbreitung und Ausstrahlung der delischen Reliefs über die Kykladen hinaus s. Schmidt (1991) 38 f. mit Anm. 225.

98 Grundlegend: Couilloud (1974); Schmidt (1991) mit Rezensionen Gnomon 64/65, 1994, 468 ff. (A. Linfert); BJb 196, 1996,756 f. (H.-H. v. Prittwitz und Gaffon). Leider bislang unpubliziert und der Verf. nicht zugänglich: D. W. v. Moock, Selbstdarstellung delischer Bürger auf hellenistischen Grabmälern von Rheneia (ungedr. Mag. München 1989), A. Prinn, Hellenistische und römische Grabreliefs von den griechischen Inseln (ungedr. Diss. Mainz 1997).

${ }^{99}$ s. Schmidt (1991) bes. 35 ff.

${ }_{100}$ Zur Dexiosis in Bezug auf Grabreliefs s. grundlegend G. Neumann, Gesten und Gebärden in der griechischen Kunst (1965) 49 ff. bes. 57 f. (S. 58: »Der Händedruck ist gewissermaßen ein Siegel der Zusammengehörigkeit und Verbundenheit, sei es der Lebenden und Verstorbenen oder nur dieser.«); B. Schmaltz, Griechische Grabreliefs (1983) 207 ff.; Zusammenfassung des Forschungsstandes mit Bibliographie: A. Scholl, Die attischen Bildfeldstelen des 4. Jhs. v. Chr. Untersuchungen zu den kleinformatigen Grabreliefs im spätklassischen Athen, 17. Beih. AM (1996) 164 ff.; für attische Grabreliefs s. zuletzt N. Sojc, Trauer auf attischen Grabreliefs. Frauendarstellungen zwischen Ideal und Wirklichkeit (2005) 120 ff. Zur Bedeutung der Dexiosis zwischen einem sitzenden Mann und einer stehenden Frau auf Grabreliefs vgl. M. Cremer, Die Dexiosis auf hellenistischen Grabstelen, in: Studien zum antiken Kleinasien III, Asia Minor Studien 16 (1995) 1 ff. 
Inseln: So sind auf Samos und Rhodos vor allem Darstellungen von Totenmahlen beliebt ${ }^{101}$, ebenso wie in Mysien und Bithynien ${ }^{102}$.

Zur allgemeinen Komposition mit zwei einander gegenüber sitzenden und einer mittig stehenden Figur lassen sich vergleichbare Szenen auf Grabreliefs in Kyzikos finden. Hier rahmen sitzende Ehepaare einen in der Mitte stehenden Jüngling ${ }^{103}$, welcher zumeist als Hauptperson anzusprechen ist. Die Reliefs datieren in das 1. Jahrhundert v. Chr. bzw. in die Kaiserzeit ${ }^{104}$, wobei Dexiosis-Szenen aus Kyzikos jedoch nicht bekannt sind $^{105}$. In Bezug auf die Verteilung der männlichen und weiblichen Figuren ist ein Relief aus Delos interessant, auf dem zwei sitzende Frauen und ein in der Mitte stehender Jüngling dargestellt sind ${ }^{106}$; ferner befindet sich neben der am rechten Bildrand Sitzenden eine kleine Dienerin, die andere Frau hält ein Kind auf dem Schoß, ein weiteres steht zu ihrer linken Seite. Das Relief wird an das Ende des 2. bzw. an den Beginn des 1. Jahrhunderts v. Chr. gesetzt ${ }^{107}$. Bei den samischen Totenmahlreliefs finden sich auch sehr häufig zwei einander gegenüber sitzende Frauen, die das Geschehen in der Mitte der Darstellung rahmen ${ }^{108}$. Alle bisher genannten Reliefs zeigen jedoch keinen Handschlag der mittigen Figur mit einer der Sitzenden.

E. Pfuhl und H. Möbius führen den Typ »Sitzende Frau im Handschlag mit einem oder einer Stehenden «109 an. Nur wenige Darstellungen zeigen eine stehende männliche Figur und eine zu ihrer linken Seite sitzende ${ }^{110}$. Die Zusammensetzung der Gesamtkomposition des ephesischen Reliefs aus unterschiedlichen Einzelkomponenten - ohne auf ein festes Schema zurückzugreifen - zeigt, dass es sich um eine sehr individuelle Darstellung handelt, die im Hellenismus in den verschiedensten Regionen zu unterschiedlichen Zeiten auftritt. Das Motiv der Dexiosis ist bevorzugt auf Delos verwendet worden. Gerade bei Betrachtung des erwähnten Reliefs aus Delos ${ }^{111}$ mit den axialsymmetrisch sitzenden Frauen, die jedoch nicht unterschiedlich hervorgehoben sind, und dem Jüngling fallen bestimmte kompositorische Einflüsse auf.

Eine besondere Beachtung sollten darüber hinaus die Dienerinnen ${ }^{12}$ des ephesischen Reliefs finden, die ja allgemein auf den gesellschaftlichen Status der Frauen, denen sie zugeordnet sind, hinweisen. Eine Abstraktion durch die sonst geläufige starke Verkleinerung ${ }^{113}$ wurde vermieden. Sie sind auffallend groß und $»$ im Verhältnis zu den Hauptpersonen realistisch proportioniert « ${ }^{114}$ dargestellt. Dieses Phänomen findet sich auf samischen Totenmahlreliefs, auf welchen auch ihr Platz stets hinter dem Stuhl der Herrin ist, noch bis in das 1. Jahrhundert v. Chr. ${ }^{115}$.

Mit dem neugefundenen Grabrelief aus der Nekropole am Ostabhang des Panayır Dağ hat sich ein sehr individuelles Familienbildnis erhalten. Es handelt sich nicht um übliche Schemata, die für hellenistische Grabreliefs in den einzelnen Landschaften im Allgemeinen bevorzugt werden, die Komposition der Figuren hat sich als singulär herausgestellt und unterliegt somit keiner bekannten zeitgleichen serienmäßigen Produktion. Einflüsse von Samos - hier sei an die Größe und Position der Dienerinnen erinnert - und vor allem

101 Fabricius (1999).

102 Cremer (1991); M. Cremer, Hellenistisch-römische Grabstelen im nordwestlcihen Kleinasien, 2. Bithynien, Asia Minor Studien 4, 2 (1992). Ferner zu den Grabstelen aus diesem Gebiet s. Schmidt (1991) 24 ff.

103 Vgl. Grabstelen aus Mysien (Kyzikos), bei denen das verstorbene Kind in der Mitte der sitzenden Eltern dargestellt ist: Cremer (1991) 59. 137 f. KH 9 Taf. 6 (Grabstele für Menophantes) = E. Schwertheim, Die Inschriften von Kyzikos und Umgebung I, Inschriften griechischer Städte aus Kleinasien 18, 1 (1980): Grabtexte Nr. 522 Taf. 38 ; S. 51.133 KN 23 Taf. 5 (Grabstele des Menophanes) $=$ Schwertheim a. O. Nr. 337 Taf. 27. Weiters Grabstele für Apollonios: Schwertheim a. O. Nr. 50 Taf. 3 , bei Pfuhl - Möbius I 275 Nr. 1110 Taf. 167 mit noch unbekannter Provenienz.

104 Datierungen s. bei den jeweiligen bibliographischen Angaben in Anm. 103.

105 Schmidt (1991) 31.

106 Couilloud (1974) 130 f. Nr. 187 Taf. 44.

107 Datierung nach Couilloud (1974) $130 \mathrm{f}$.

108 Fabricius (1999) $115 \mathrm{f}$.

109 Pfuhl - Möbius I 262 ff. Nr. 1050 ff.

110 Von Syme (2. Jh.): Pfuhl - Möbius 267 Nr. 1073 Taf. 160. Von Chios (1. Jh.): Pfuhl - Möbius I 267 Nr. 1074 Taf. 161. Aus Sardes (Ende 3. Jh.): Pfuhl - Möbius I 267 Nr. 1072 Taf. 161. Aus Istanbul (3. Jh.): Pfuhl - Möbius I 272 Nr. 1100 Taf. 166. Zu Byzantion s. ferner Schmidt (1991) 31.

111 Couilloud (1974) 130 f. Nr. 187 Taf. 44, wobei sich die Grabepigramme auf die beiden Frauen beziehen.

112 Zu Dienerdarstellungen s. Bibliographie bei Fabricius (1999) 92 Anm. 72.

113 Pfanner (1989) 174.

114 Fabricius (1999) 93

115 Fabricius (1999) 118. 
von Delos (Dexiosis) sind evident. Auf Delos findet sich eine bewusste Widerspiegelung »der klassischen attischen Grabkunst in zeitgenössischen Formen ${ }^{116}$. Handschlagszenen treten gehäuft auf, und die Bilder sind auf die Darstellung der Personen beschränkt: Architekturangaben, Altäre, Bäume und dergleichen fehlen bei den delischen Reliefs völlig.

Im stilistischen Vergleich mit den Grabreliefs von Delos kann die Datierung der ephesischen Reliefs genauer eingegrenzt werden. St. Schmidt unterteilte die delischen Reliefs, die in einem Zeitraum von $166 \mathrm{v}$. Chr. bis 88 bzw. 69 v. Chr. entstanden sind ${ }^{117}$, in fünf zeitlich aufeinander abfolgende Gruppen. An diese Gruppen versuchte er, die weiteren bekannten Reliefs aus Byzantion und Kyzikos anzuschließen ${ }^{118}$.

Da es sich bei dem neugefundenen Relief um eine Arbeit von handwerklich einfachem Niveau handelt, wird eine stilistische Untersuchung naturgemäß erschwert. Die allgemeinen Mängel in der Ausführung liegen in missglückten Körperdrehungen - man denke an die Haltung des Jünglings oder an die Sitzende vor ihm - und Verkürzungen von Gliedmaßen - hier sei an den linken Arm der Sitzenden im Handschlag erinnert. Zusätzlich ist die Ausführung der Oberfläche stellenweise unfertig, was eine stark reduzierte Plastizität bedingt. St. Schmidt konnte in seiner Untersuchung zeigen, dass trotz der 'handwerklichen' Reliefs von Delos allgemeine stilistische Eigenheiten zutreffen ${ }^{119}$. Da unser Relief ikonographisch deutlich von den delischen Reliefs abhängig ist, scheint es legitim, es auf diese allgemeinen Charakteristika hin zu überprüfen.

Bei der Sitzenden im Typus der Pudicitia fällt die parallele Beinhaltung auf, wie sie bei den Sitzenden der ersten drei Gruppen nach Schmidt nicht vorkommt. Die aufrechte Haltung der Sitzenden bildet sich ebenso im Laufe der Entwicklung heraus ${ }^{120}$. Deutliche Gemeinsamkeiten zeigen sich mit der Gruppe IV nach Schmidt, bei der die gesamte Figur einer »fließenden Gesamtform « unterliegt, »der alle Gewand- und Körperangaben untergeordnet sind ${ }^{121}{ }^{12}$. Ferner ist es die $»$ vereinheitlichte Oberflächengestaltung $\aleph^{122}$, gepaart mit der unstofflichen Darstellung der Gewandung ${ }^{123}$ : So werden auch die Falten als Linienwerte verwendet ${ }^{124}$.

Auch wenn die vorgeschlagenen Datierungen von St. Schmidt nicht unwidersprochen blieben ${ }^{125}$, so erlauben sie dennoch eine grobe zeitliche Eingrenzung des Reliefs vom Ostabhang des Panayır Dağ. Folgt man der chronologischen Reihe der Grabdenkmäler von Delos, so ergibt sich eine Datierung kurz vor oder kurz nach 100 v. Chr.

J. Auinger

\section{Abgekürzt zitierte Literatur}

\begin{tabular}{|c|c|}
\hline Atalay (1973) & Atalay, Ein späthellenistisches Grabrelief aus Ephesos, AA 1973, $231 \mathrm{ff}$. \\
\hline Atalay (1988) & E. Atalay, Hellenistik çag'da Ephesos Mezar Stelleri Atölyeleri (1988). \\
\hline Berns (2003) & $\begin{array}{l}\text { Ch. Berns, Untersuchungen zu den Grabbauten der frühen Kaiserzeit in Kleinasien, Asia Minor Stu- } \\
\text { dien } 51 \text { (2003). }\end{array}$ \\
\hline Couilloud (1974) & M.-Th. Couilloud, Les Monuments funéraires de Rénée, Délos XXX (1974). \\
\hline Cremer (1991) & $\begin{array}{l}\text { M. Cremer, Hellenistisch-römische Grabstelen im nordwestlichen Kleinasien, 1. Mysien, Asia Minor } \\
\text { Studien 4, } 1 \text { (1991). }\end{array}$ \\
\hline Fabricius (1999) & $\begin{array}{l}\text { J. Fabricius, Die hellenistischen Totenmahlreliefs. Grabpräsentation und Wertvorstellungen in ostgrie- } \\
\text { chischen Städten (1999). }\end{array}$ \\
\hline IvE & Inschriften griechischer Städte aus Kleinasien 11, 1 (1979) - 17, 4 (1984); Ephesos (IvE Ia-VII 4). \\
\hline Kabus-Preisshofen (1989) & R. Kabus-Preisshofen, Die hellenistische Plastik der Insel Kos, 14. Beih. AM (1989). \\
\hline Knibbe - Langmann (1993) & D. Knibbe - G. Langmann, Via sacra Ephesiaca 1, BerMatÖAI 3 (1993). \\
\hline
\end{tabular}

\footnotetext{
116 Schmidt (1991) 42.

117 Schmidt (1991) 65 f. Zu den Gruppen vgl. ebenda Tab. 1.

118 Schmidt (1991) Tab. 3. 4.

$119 \mathrm{Zu}$ dem Problem der stilistischen Untersuchung bei handwerklich einfachen Arbeiten vgl. Schmidt (1991) $66 \mathrm{f}$.

120 Schmidt (1991) 71.

121 Schmidt (1991) 70.

122 Schmidt (1991) 70.

123 Schmidt (1991) 75.

124 Schmidt (1991) 71.

${ }^{125}$ Linfert (Anm. 98) 469.
} 
Pfanner (1989)

Pietsch (1999)

Schmidt (1991)

Thür (1999)

Wood (1877)
M. Pfanner, Ein Relief in der Münchner Glyptothek und Überlegungen zu einigen bemerkenswerten Aspekten hellenistischer Grabreliefs, AM 104, 1989, $165 \mathrm{ff}$.

W. Pietsch, Außerstädtische Grabanlagen von Ephesos, in: H. Friesinger - F. Krinzinger (Hrsg.), 100 Jahre Österreichische Forschungen in Ephesos. Akten des Symposions Wien 1995, AForsch 1, DenkschrWien 260 (1999) 455 ff.

St. Schmidt, Hellenistische Grabreliefs. Typologische und chronologische Beobachtungen (1991).

H. Thür, Via Sacra Ephesiaca. Vor der Stadt und in der Stadt, in: P. Scherrer - H. Taeuber - H. Thür (Hrsg.), Steine und Wege. Festschrift D. Knibbe, SoSchrÖAI 32 (1999) 163 ff.

J. T. Wood, Discoveries at Ephesus (1877).

\section{Dr. Alexander Sokolicek}

Dr. Johanna Auinger

co Österreichische Archäologisches Institut, Franz Klein-Gasse 1, A-1190 Wien

E-Mail: vorname.nachname@oeai.at

Abbildungsnachweis: Abb. 1: Ch. Kurtze; Abb. 2-6: A. Sokolicek; Abb. 7-11: N. Gail; Abb. 12: J. Auinger; alle Abbildungen (C) ÖAI. 